\title{
OPEN Liraglutide, a glucagon-like peptide 1 receptor agonist, exerts analgesic, anti-inflammatory and anti-degradative actions in osteoarthritis
}

\author{
C. Meurot ${ }^{1}$, C. Martin ${ }^{1}$, L. Sudre ${ }^{1}$, J. Breton ${ }^{1}$, C. Bougault ${ }^{2}$, R. Rattenbach ${ }^{1,3}$, K. Bismuth ${ }^{1}$, \\ C. Jacques ${ }^{2} \&$ F. Berenbaum ${ }^{3,4 \bowtie}$
}

Osteoarthritis (OA) is a common disabling disease worldwide, with no effective and safe diseasemodifying drugs (DMOAD) in the market. However, studies suggest that drugs, such as liraglutide, which possess strong potential in decreasing low-grade systemic inflammation may be effective in treating OA. Therefore, the aim of this study was to examine the anti-inflammatory, analgesic, and anti-degradative effects in $\mathrm{OA}$ using in vitro and in vivo experiments. The results showed that intraarticular injection of liraglutide alleviated pain-related behavior in in vivo sodium monoiodoacetate OA mouse model, which was probably driven by the GLP-1R-mediated anti-inflammatory activity of liraglutide. Moreover, liraglutide treatment significantly decreased IL-6, $\mathrm{PGE}_{2}$ and nitric oxide secretion, and the expression of inflammatory genes in vitro in chondrocytes and macrophages in a dose-dependent manner. Additionally, liraglutide shifted polarized macrophage phenotype in vitro from the pro-inflammatory M1 phenotype to the M2 anti-inflammatory phenotype. Furthermore, liraglutide exerted anti-catabolic activity by significantly decreasing the activities of metalloproteinases and aggrecanases, a family of catabolic enzymes involved in cartilage breakdown in vitro. Overall, the findings of this study showed that liraglutide ameliorated OA-associated pain, possess anti-inflammatory and analgesic properties, and could constitute a novel therapeutic candidate for OA treatment.

Osteoarthritis (OA) is a prevalent disabling disease, affecting over 300 million individuals worldwide ${ }^{1-3}$. Owing to the pain and loss of function induced by this disease, it reduces the quality of life and causes a considerable economic burden estimated at $\$ 350$ billion in the United States alone ${ }^{4}$. Additionally, because of the sedentary lifestyle associated with OA, it increases cardiovascular mortality by more than $50 \%$, necessitating its listing in 2018 as a severe disease by the Food and Drug Administration (FDA) ${ }^{5}$. To date, there is no effective treatment for the disease, except for a few symptomatic drugs that are often weakly effective or poorly tolerated ${ }^{6}$.

$\mathrm{OA}$ is characterized by joint damage, affecting mainly the cartilage, synovial membrane, and subchondral bone. Synovitis, inflammation of the synovium, can occur in the early stages of $\mathrm{OA}^{7,8}$. OA synovitis directly contributes to several clinical signs and symptoms, including joint swelling and effusion, and reflects the structural progression of the disease $e^{8,9}$. Therefore, treatment capable of reducing synovial inflammation and slowing down cartilage destruction should have the essential characteristics of disease-modifying OA drugs (DMOADs).

In the early stage, inflammation leads to the secretion of inflammatory and pro-degradative mediators, including cytokines such as interleukin- $1 \beta$ (IL-1 $\beta$ ), interleukin-6 (IL-6), and tumor necrosis factor- $\alpha$ (TNF$\alpha$ ), lipid mediators such as prostaglandin E2 $\left(\mathrm{PGE}_{2}\right)$, and matrix metalloproteinases (MMPs) by macrophages and fibroblast-like synoviocytes ${ }^{7,10}$. These mediators can induce the production of MMPs and a disintegrin and metalloproteinase with thrombospondin motifs (ADAMTS) by chondrocytes and further degradation of the cartilage, contributing to a cycle of inflammation and disease $\mathrm{e}^{7,10}$.

${ }^{1} 4$ P-Pharma, Lille, France. ${ }^{2}$ Sorbonne University, INSERM CRSA St-Antoine, Paris, France. ${ }^{3}$ MMoving Biotech, Lille, France. ${ }^{4}$ Sorbonne University, INSERM CRSA, Rheumatology Department, AP-HP St-Antoine, Paris, France. ${ }^{\varpi}$ email: francis.berenbaum@aphp.fr 
Moreover, macrophages are the main immune cell type in healthy synovium and are likely the front-line cells that sense joint damage. These cells also contribute to OA progression by producing MMPs and cytokines ${ }^{11}$. Studies have shown that macrophages accumulate and become polarized towards the M1 phenotype in the synovium during OA development ${ }^{12}$.

Among the explanations for joint inflammation observed during OA is the hypothesis that the low-grade systemic inflammation observed during metabolic diseases could be one of the factors initiating and aggravating the OA process ${ }^{13}$. Several mechanisms have been implicated in the pathophysiology of diabetes-induced OA. Chronic hyperglycemia results in the accumulation of advanced glycation end products (AGEs), which is higher in diabetic patients than in normal patients ${ }^{14}$. AGEs activate inflammatory and oxidative pathways within the joint tissue ${ }^{15}$. Furthermore, hyperglycemia exerts synergistic effects with IL- $1 \beta$ and can directly increase oxidative stress and inflammation. Thus, hyperglycemia may increase responsiveness to local low-grade inflammation ${ }^{16,17}$. We hypothesized that drugs that have previously demonstrated strong potential in decreasing low-grade systemic inflammation could also act locally in the joint. Glucagon-like peptide-1 (GLP-1) is an incretin, a hormone secreted by the gut during meals that triggers the pancreas to produce insulin, which possesses a spectrum of extra-pancreatic functions related to its anti-inflammatory properties ${ }^{18-20}$. Liraglutide, commercially known as Victoza, is a modified human GLP-1(7-37) with a longer half-life and is administered for type II diabetes ${ }^{21}$. GLP-1 receptor (GLP-1R) is expressed in pancreatic islets as well as in several extra-pancreatic organs or cell lineages, indicating that GLP-1-based drugs can exert extra-pancreatic functions. GLP-1 shows anti-inflammatory properties in pancreatic islets and adipose tissue, contributing to lower glucose levels in diabetic patients ${ }^{22,23}$. In addition to these tissues, emerging data suggest that GLP-1-based therapies possess anti-inflammatory effects on the liver, brain, kidney, lung, testis, skin, and vascular system including aorta and vein endothelial cells, by reducing the production of inflammatory cytokines and infiltration of immune cells in the tissues ${ }^{19,24}$. Thus, we speculated that GLP-1-based therapies, especially liraglutide ${ }^{25}$, may be beneficial for the treatment of OA due to its anti-inflammatory and anti-catabolic effects as well as its potential analgesic properties when injected intraarticularly. However, the anti-inflammatory mechanism of liraglutide is poorly understood.

Therefore, the aim of this study was to examine the analgesic and anti-inflammatory properties of liraglutide in treating OA, using in vivo and in vitro experiments. The findings of this study could provide potential therapeutic materials or target for treating OA. Here, we show here that GLP-1R is expressed in the cartilage and synovium in human diseased and mouse normal joint tissues. Moreover, we demonstrated that intra-articular (IA) liraglutide alleviates pain-related behavior in a sodium monoiodoacetate (MIA) OA mouse model displaying an analgesic effect. This analgesic effect observed in vivo is likely underlain by liraglutide's concerted antiinflammatory action, as demonstrated in vivo in a mouse model of MIA, whereby liraglutide improves synovitis severity score, and also in vitro, whereby liraglutide dose-dependently decreased $\mathrm{PGE}_{2}$, nitric oxide (NO), and IL-6 secretion, along with iNos, Cox2, and Tnf- $\alpha$ gene expression levels in both chondrocytes and macrophages. In addition, liraglutide is able in vitro to shift the polarized macrophage phenotype from the pro-inflammatory M1 phenotype to the M2 anti-inflammatory phenotype. The ability of the GLP-1R antagonist, exendin 9-39, to compete with away liraglutide's anti-inflammatory effects, confirmed that GLP-1R is the primary target of liraglutide in chondrocytes and macrophages. Finally, our in vitro results indicated that liraglutide may attenuate cartilage degradation through an anti-catabolic effect at both at the protein and gene expression levels. Targeting inflammation and cartilage breakdown in two main cellular actors of the diseased joint, and alleviating pain in vivo, confers to liraglutide the properties of a potential disease modifier, which could constitute a new treatment for OA.

\section{Results}

GLP-1 receptor is expressed in articular cartilage and synovial membrane in human and mice. Immunohistochemical examination of superficial and intermediate layer cartilage sections of human OA knee joints indicated that chondrocytes express the GLP-1R protein (Fig. 1a). Additionally, positive GLP-1R staining was observed in human synovial membrane sections from OA patients, specifically at the intima and blood vessel levels (Fig. 1b). Furthermore, immunohistochemical analysis of the knee joints of mouse indicated positive GLP-1R staining in the tibia and femoral articular cartilage, meniscus, and bone marrow of healthy murine knee articular sections (Fig. 1c). Similarly, a cluster of synovial cells showed positive staining for GLP-1R in healthy murine synovial membrane (Fig. 1d). Overall, these results suggest a central and pleiotropic potential role of GLP-1R in normal and diseased knee joints from human and mouse species. Expression of GLP1-R was confirmed by RT-qPCR analysis of RNA from primary human chondrocytes from cartilage explants from 18 human OA patients (data not shown).

Liraglutide exerts analgesic and anti-inflammatory effects in vivo in a short-term sodium monoiodoacetate murine model of osteoarthritis. The effect of intra-articular injection of liraglutide was evaluated in vivo using short-term (up to 10 days) MIA OA mice model. The effect of liraglutide on OA-associated pain was assessed using the von Frey test. In this model, behavioral experiments were performed on day 2 (randomization), 7 and 10. Following IA injection of MIA, mice exhibited a significant decrease in paw withdrawal threshold as early as day $2(p<0.0001)$ compared to saline-treated mice and the difference in mechanical allodynia remained statistically significant until the end of the experiment (Fig. 2a). On day 3, that is 2 days after MIA model induction, a single IA injection of liraglutide induced a significant dose-dependent increase in paw withdrawal threshold compared with the vehicle-treated group on day 7 (liraglutide $5 \mu \mathrm{g} p<0.0001$, liraglutide $10 \mu \mathrm{g} p=0.0002$, and liraglutide $20 \mu \mathrm{g} p=0.0011$ ), with $20 \mu \mathrm{g}$ of liraglutide having similar effects with $20 \mu \mathrm{g}$ of dexamethasone (positive control, $p=0.0022$ ). Similarly, there was a significant dose-dependent increase in paw withdrawal threshold in the liraglutide-treated groups compared with the vehicle group on day 
a
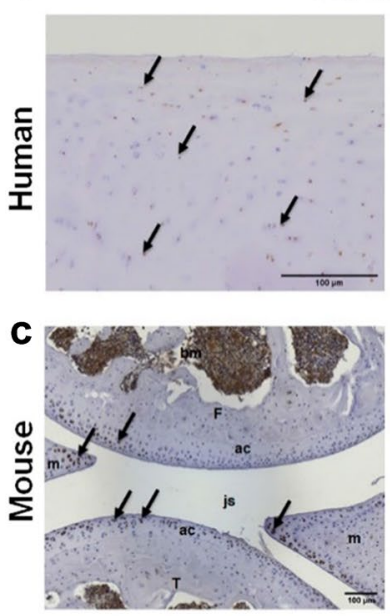

Cartilage
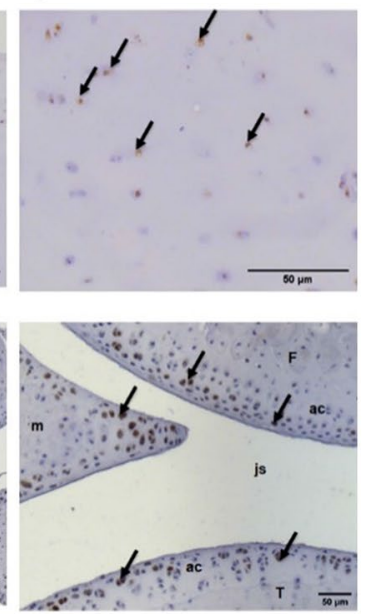

b
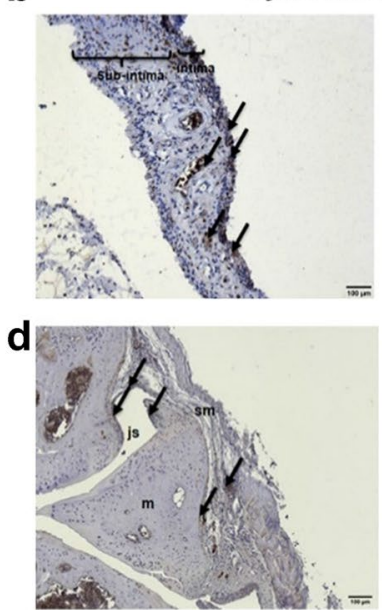

Synovial membrane
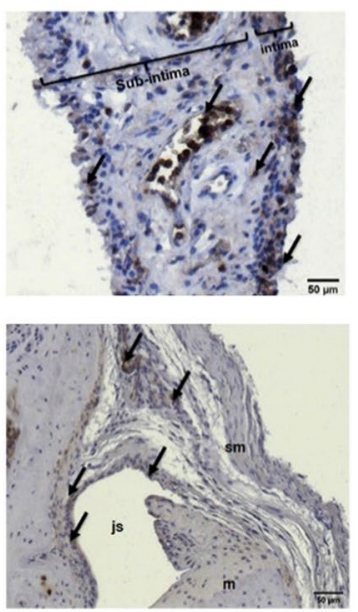

Figure 1. Expression of GLP-1 receptor in OA human and non-OA mouse knee joint. (a) Immunohistochemical staining of human OA knee cartilage sections was performed to determine the presence of GLP-1R (Mankin score: 3/14, scale bar $=100 \mu \mathrm{m}$ or $50 \mu \mathrm{m}$ ). (b) Immunohistochemical staining of human OA synovial membrane sections was performed to determine the presence of GLP-1R (si: subintima, $i$ : intima, $v$ : blood vessel, scale bar $=100 \mu \mathrm{m}$ or $50 \mu \mathrm{m}$ ). (c) Immunohistochemical staining of non-OA mice knee joint sagittal sections to determine the presence of GLP-1R (ac: articular cartilage, $m$ : meniscus, js: joint space, $T$ : tibia, $F$ : femur, $b m$ : bone marrow, scale bar $=100 \mu \mathrm{m}$ or $50 \mu \mathrm{m}$ ). (d) Immunohistochemical staining of non-OA mice synovial membrane sagittal sections to determine the presence of GLP-1R ( $s m$ : synovial membrane, $j$ s: joint section, $m$ : meniscus, scale bar $=100 \mu \mathrm{m}$ or $50 \mu \mathrm{m}$ ). Control experiment was performed without primary antibody incubation. Arrows indicate example of cells positive for GLP-1R staining.
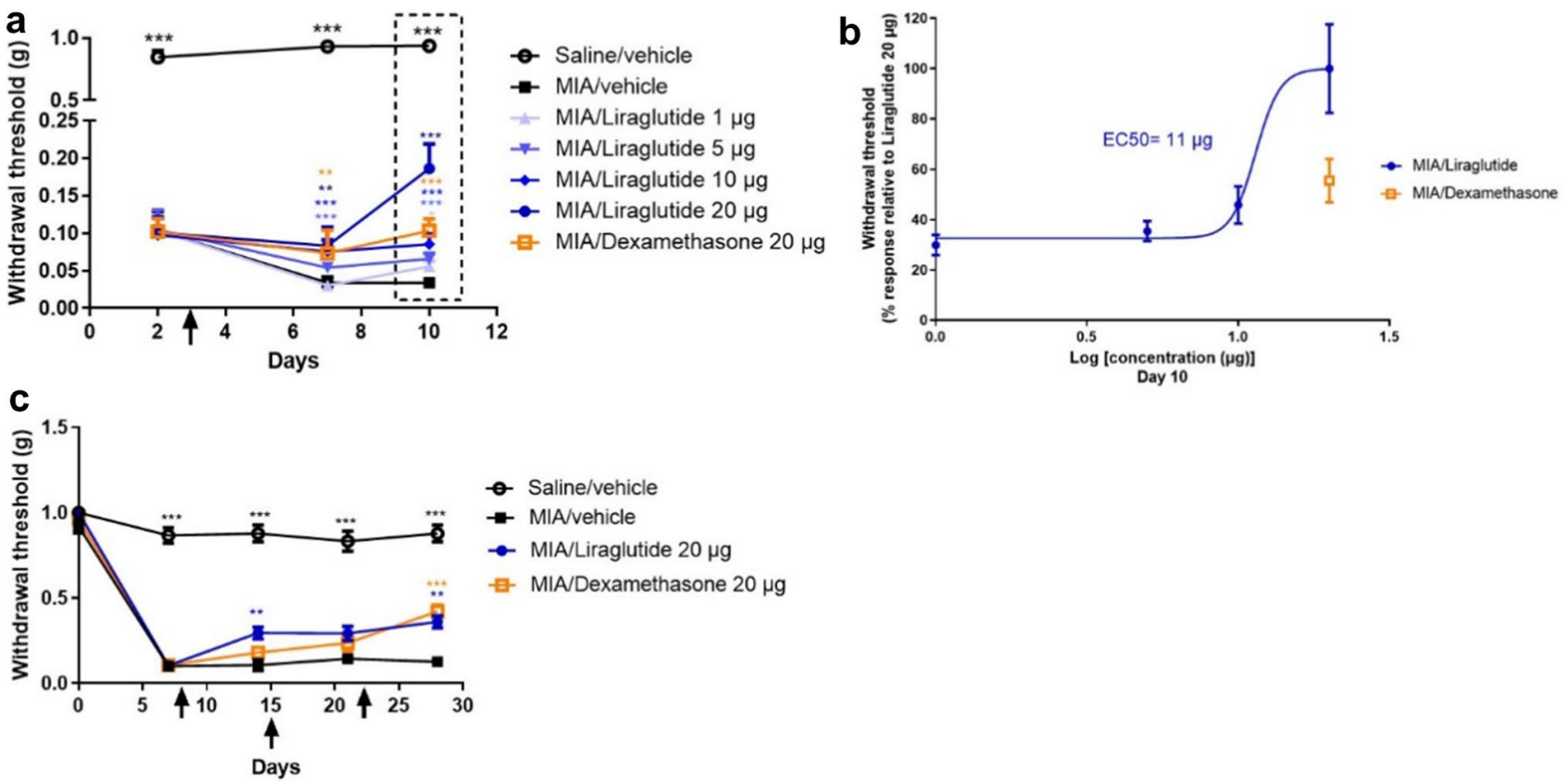

Figure 2. Liraglutide displayed analgesic effect in MIA mice models of OA. Mice knee joints were intraarticularly (IA) injected with $0.75 \mathrm{mg}$ of MIA or saline on day 1 . For the short-term study (a,b), treatments (liraglutide, dexamethasone, or vehicle) were injected IA on day 3 and inflammation pain sensitivity was determined by the von Frey test on day 2 (for randomization), 7, and 10 ( $n=15-19$ per group). For the longterm study (c), treatments were administered on days 8, 15, and 22, and von Frey tests were performed on day 7 (for randomization), 14, 21, and 28 ( $\mathrm{n}=9-10$ per group). (a) Paw withdrawal threshold was assessed by von Frey filament stimulation on days 2, 7, and 10. (b) The efficacy rate of liraglutide in the MIA short-term study was analyzed using GraphPad Prism 9.0 and the $\mathrm{EC}_{50}$ value was determined at day 10 from the von Frey calculated values. (c) Paw withdrawal threshold was assessed by von Frey filament stimulation on days 0, 7, 14, 21, and 28. Arrows indicate the treatment with IA administration. Statistical analysis: Mean \pm SEM. Mann-Whitney test with sequential strategy, ${ }^{* *} p<0.01,{ }^{* * *} p<0.001,{ }^{* * *} p<0.0001$ versus MIA control. 
a

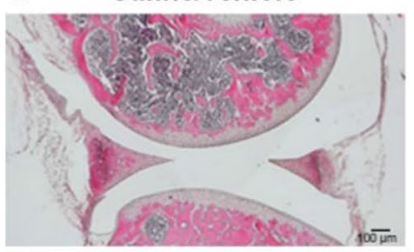

b

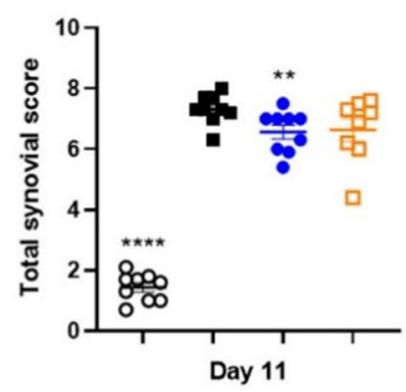

MIA/vehicle

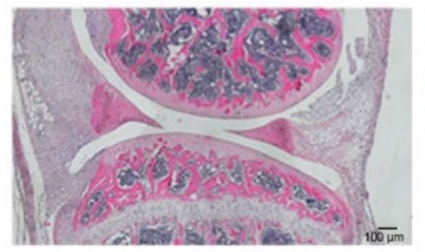

- Saline/vehicle

- MIA/vehicle

- MIA/Liraglutide $20 \mu \mathrm{g}$

口 MIA/Dexamethasone $20 \mu \mathrm{g}$
MIA/Liraglutide $20 \mu \mathrm{g}$

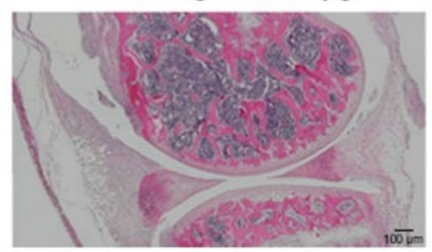

C

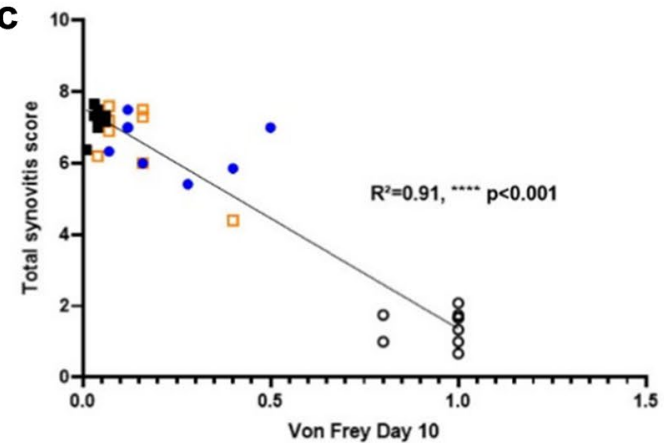

Figure 3. Liraglutide displayed anti-inflammatory effect in vivo in short-term MIA mice model of OA. Mice knee joints were intra-articularly (IA) injected with $0.75 \mathrm{mg}$ of MIA or saline on day 1 . Treatments (liraglutide, dexamethasone, or vehicle) were IA injected on day 3 ( $n=15-19$ per group). At the end of the study, on day 11, the mice were euthanized, and the right knee joint was recovered for histological analyses. (a) Representative photographs of sagittal sections of the paws of mice IA injected with $0.75 \mathrm{mg}$ of MIA to induce inflammation and subsequently IA treated with vehicle, liraglutide or dexamethasone (positive control). (b) Histogram representing the total synovitis score calculated from Krenn et al., synovitis score system ( $n=8-9$ per group). (c) Based on the results of the von Frey test on day 10 and the synovitis score obtained for each animal, a correlation curve between these two parameters was calculated using GraphPad Prism $9.0(n=8-9$ per group). Statistical analysis: Mean \pm SEM. Mann-Whitney test with sequential strategy, ${ }^{* *} p<0.01,{ }^{* * *} p<0.0001$ versus MIA control. Simple linear regression, ${ }^{* * *} p<0.0001$.

10 (liraglutide $1 \mu \mathrm{g} p=0.0196$, liraglutide $5 \mu \mathrm{g} p=0.0004$, and liraglutide $10 \mu \mathrm{g}$ and $20 \mu \mathrm{g} p<0.0001$, compared with the vehicle-treated group). Notably, the efficacy of liraglutide $20 \mu \mathrm{g}$ was superior to that of dexamethasone $(p<0.0001)$ at day 10. The calculated median effective concentration $\left(\mathrm{EC}_{50}\right)$ was $11 \mu \mathrm{g}$ (Fig. $\left.2 \mathrm{~b}\right)$. Based on the results of the short-term model, $20 \mu \mathrm{g}$ liraglutide was selected for histopathological analyses (Fig. 3). Liraglutide treatment significantly improved synovitis severity score in the MIA mice model (saline/vehicle $p<0.0001$, liraglutide $20 \mu \mathrm{g} p=0.0099$, compared to MIA/vehicle-treated group) (Fig. 3a,b). Although dexamethasone $20 \mu \mathrm{g}$ also reduced the total Krenn score ${ }^{26}$, its effect was not significant (dexamethasone $20 \mu \mathrm{g} p=0.1288$ ) (Fig. 3b). Furthermore, correlation analysis between synovitis score (Fig. 3b) and the results of the von Frey test at day 10 (Fig. 2a,b) indicated $91 \%$ correlation $\left(\mathrm{R}^{2}=0.91 p<0.0001\right)$ between inflammation and pain. Overall, liraglutide treatment successfully improved the synovitis severity score in the short-term MIA mice model. The anti-pain effect observed in this study could be, at least partially, mediated by the anti-inflammatory effect of liraglutide.

Liraglutide exerts analgesic effect in vivo in a long-term sodium monoiodoacetate murine model of osteoarthritis. The effect of intra-articular injections of liraglutide were evaluated in vivo using a long-term (up to 28 days) MIA mice model. Based on the results of the short-term model, $20 \mu \mathrm{g}$ liraglutide was selected, and the potential benefit of liraglutide in OA-associated pain was assessed using the von Frey test (Fig. 2c). Behavioral experiments in repeatedly treated animals were performed on day 7 (for randomization), 14,21 , and 28. MIA injection significantly reduced the withdrawal threshold of the ipsilateral paw compared with the saline group until the end of the study. Liraglutide induced a significant improvement in mechanical allodynia over time compared with the vehicle-treated group (day 14: liraglutide $20 \mu \mathrm{g} p=0.0098$, day 28: liraglutide $20 \mu \mathrm{g} p=0.0038$, dexamethasone $20 \mu \mathrm{g} p=0.0002$, compared to vehicle-treated group).

Similar to the short-term model, $20 \mu \mathrm{g}$ of liraglutide was more efficient than dexamethasone on day 14 (liraglutide $20 \mu \mathrm{g} p=0.0098$, dexamethasone $20 \mu \mathrm{g} p=0.0568$ ). After the second and third injections, the effects of dexamethasone and liraglutide were comparable, with comparable analgesic effects at day 21 and day 28 . This clearly suggests that liraglutide acts faster than dexamethasone in relieving pain symptoms in this model.

Additionally, the treatments did not significantly affect the body weight of the animals in both the short- and long-term studies (Suppl. Fig. 1).

Taken together, these results indicated that intra-articular injection of liraglutide possess rapid analgesic effects compared to the standard treatment, and that local administration of liraglutide was well tolerated.

Furthermore, we hypothesized that the analgesic effect of liraglutide is dependent, at least partially, on its anti-inflammatory properties. To verify this assumption, an in vitro experiment was conducted using two murine cell types (chondrocytes and macrophages), which reflect two crucial cell types present in knee joints. 
a

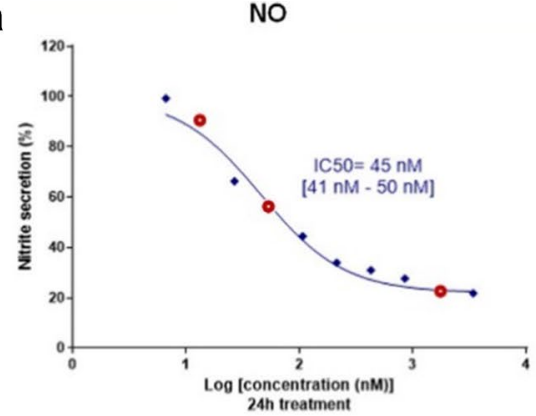

b

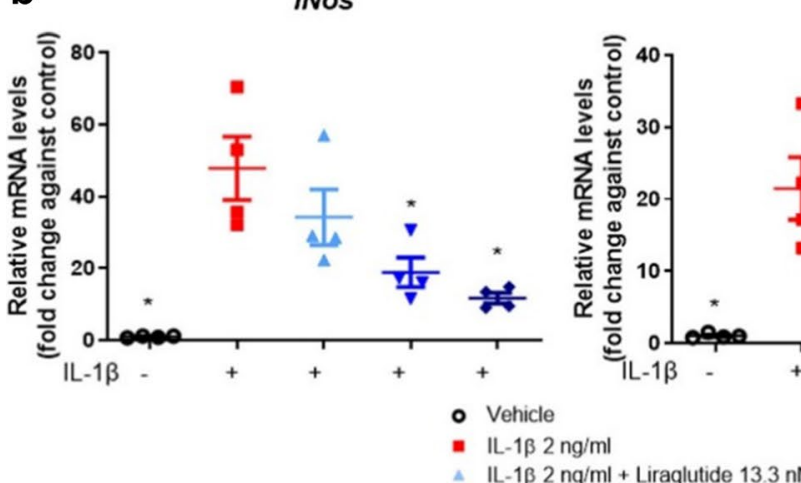

PGE2

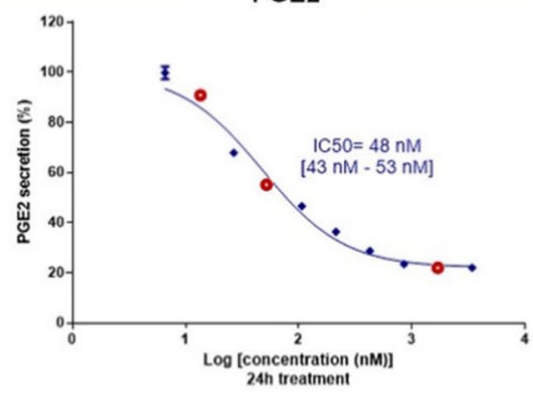

$\operatorname{Cox} 2$

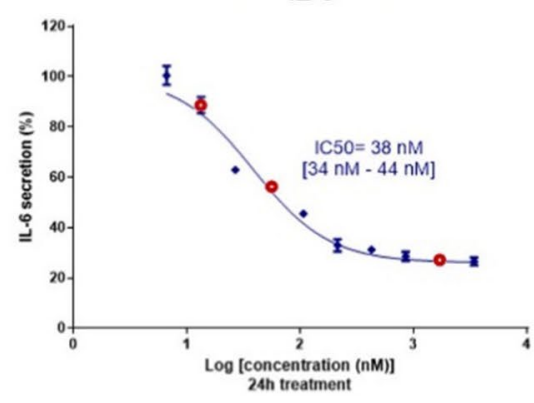

Tnf- $\alpha$

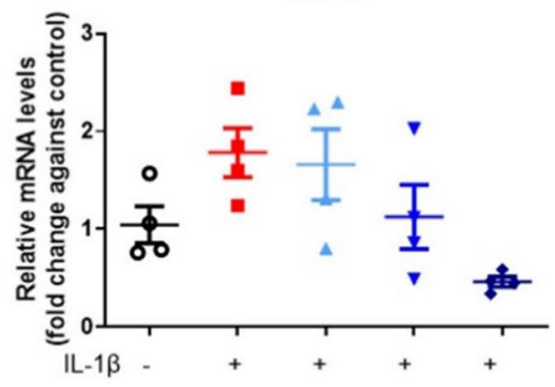

Figure 4. Anti-inflammatory effects of liraglutide in murine primary chondrocytes. Murine primary chondrocytes were stimulated with IL- $1 \beta(2 \mathrm{ng} / \mathrm{mL})$ and co-treated with 10 doses of liraglutide $(6.6,13.3,26.6$, $53.1,106.3,212.5,425,850 \mathrm{nM}, 1.7$ and $3.4 \mu \mathrm{M})$ for $24 \mathrm{~h}(\mathrm{n}=4)$. (a) The inhibition rate of liraglutide in murine primary chondrocytes was analyzed using GraphPad Prism 9.0, and the $\mathrm{IC}_{50}$ values were determined for NO, $\mathrm{PGE}_{2}$, and IL-6, which were detected in the culture supernatant. (b) Relative mRNA expression of $i$ Nos, Cox2, and Tnf- $\alpha$ in murine primary chondrocytes co-treated for $24 \mathrm{~h}$ with $2 \mathrm{ng} / \mathrm{mL} \mathrm{IL}-1 \beta$ and liraglutide ( $13.3 \mathrm{nM}$, $53.1 \mathrm{nM}$, and $1700 \mathrm{nM}$, shown in red circles in $(\mathbf{a}))$ or vehicle $(\mathrm{n}=4)$. Statistical analysis: Mean \pm SEM, MannWhitney test with sequential strategy, ${ }^{*} p<0.05$, versus stimulated control (IL-1 $\beta$ alone).

Immunohistochemical assay indicated positive GLP-1R staining at the membrane and intracellular levels in both chondrocytes and RAW 264.7 murine macrophage cell lines by immunofluorescence (Suppl. Fig. 2). Therefore, these two cell models were used to examine the potential anti-inflammatory effect of liraglutide.

Liraglutide exerts dose-dependent anti-inflammatory effect on murine primary chondrocytes. To investigate the in vitro effect of liraglutide on the production of inflammatory mediators in chondrocytes, cells were cultured with $2 \mathrm{ng} / \mathrm{mL} \mathrm{IL-1} \beta$ for $24 \mathrm{~h}$. IL-1 $\beta$-induced chondrocytes were incubated with 10 ascending doses $(6.6,13.3,26.6,53.1,106.3,212.5,425,850 \mathrm{nM}, 1.7$ and $3.4 \mu \mathrm{M})$ of liraglutide for $24 \mathrm{~h}$ to determine the half maximal inhibitory concentration $\left(\mathrm{IC}_{50}\right)$ of the inflammatory mediators $\mathrm{NO}, \mathrm{PGE}_{2}$, and IL-6 (Fig. 4a). Three doses were selected for gene expression analyses (13.3 nM, $53.1 \mathrm{nM}$, and $1700 \mathrm{nM}$, shown in red circles in Fig. 4a). Lactate dehydrogenase assay (LDH) was performed to confirm the non-cytotoxicity of liraglutide treatment on primary murine chondrocytes (data not shown). The results showed that IL-1 $\beta$ stimulation induced an increase in the secretion of the three pro-inflammatory mediators. Remarkably, liraglutide treatment significantly reduced IL-1 $\beta$-induced secretion of nitrite, $\mathrm{PGE}_{2}$, and IL-6 in a dose-dependent manner (Fig. 4a).

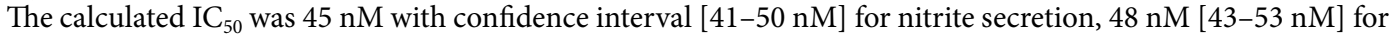
$\mathrm{PGE}_{2}$ secretion, and $38 \mathrm{nM}$ [34-44 nM] for IL-6 secretion (Fig. 4a). Similarly, liraglutide treatment significantly decreased IL-1 $\beta$-induced expression of $i$ Nos, Cox 2 and Tnf- $\alpha$ in chondrocytes ( $p=0.0286$ compared to IL-1 $\beta$ group) (Fig. 4b, Supplemental Table 1). Overall, these results demonstrated that liraglutide induced broad antiinflammatory dose-dependent effects in IL-1 $\beta$-stimulated murine chondrocytes.

Liraglutide exerts dose-dependent anti-inflammatory effect on murine macrophages. Considering the central role of macrophages in OA and the expression of GLP-1R in the macrophage cell line (Suppl. Fig. 2), the anti-inflammatory effect of liraglutide on RAW 264.7 cell line was examined. RAW 264.7 cells were cultured with $100 \mathrm{ng} / \mathrm{mL}$ of LPS at 10 ascending doses (6.6, 13.3, 26.6, 53.1, 106.3, 212.5, 425, $850 \mathrm{nM}, 1.7$ and $3.4 \mu \mathrm{M})$ of liraglutide for $24 \mathrm{~h}$ to determine the $\mathrm{IC}_{50}$ of the pro-inflammatory mediators $\mathrm{NO}, \mathrm{PGE}_{2}$, and IL-6 (Fig. 5a). Lactate dehydrogenase assay ( $\mathrm{LDH})$ was performed to confirm the non-cytotoxicity of liraglutide treatment in a murine macrophage cell line (data not shown). The results indicated that liraglutide treatment significantly reduced LPS-induced secretion of NO, PGE 2 , and IL-6 in RAW 264.7 cells in a concentrationdependent manner (Fig. 5a). The calculated $\mathrm{IC}_{50}$ was $38 \mathrm{nM}$ with confidence interval [34-44 nM] for nitrite 


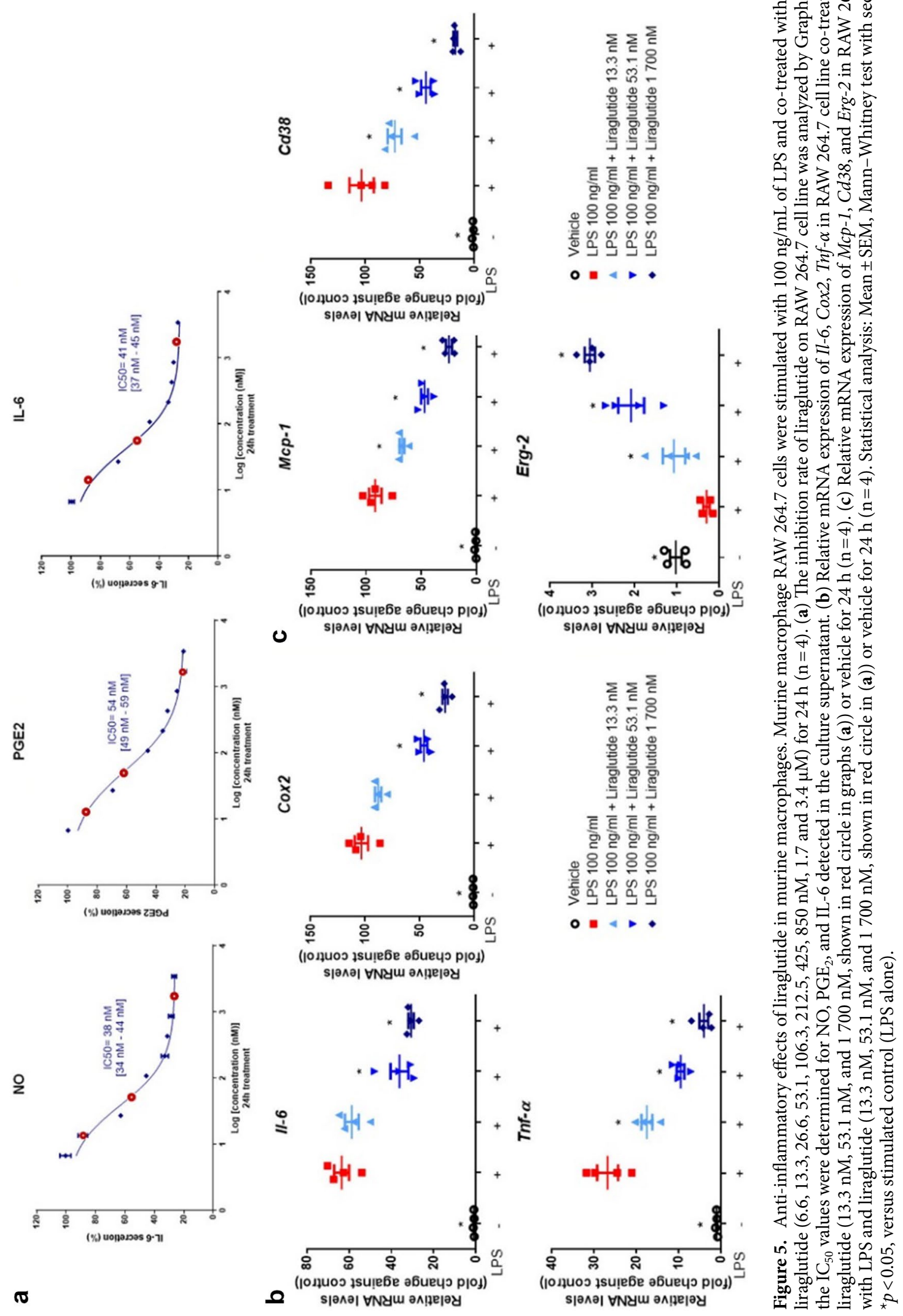


a
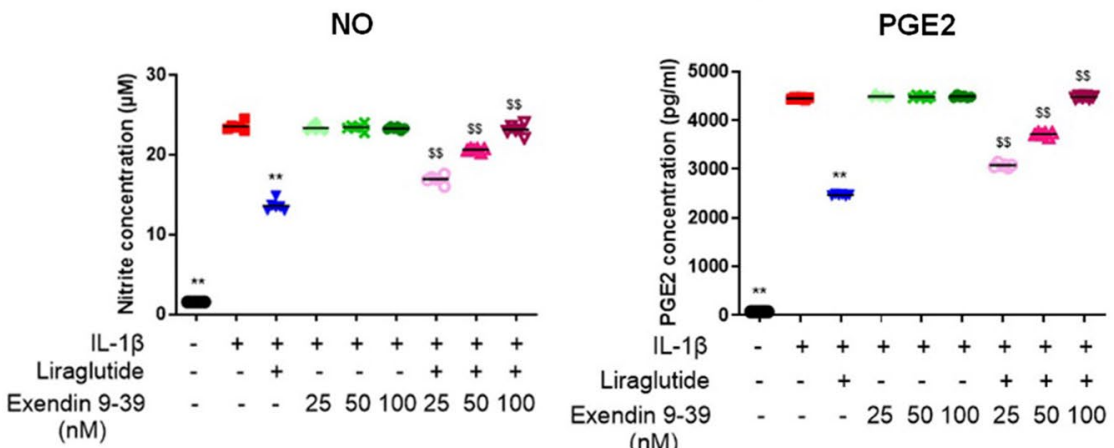

$(\mathrm{nM})$

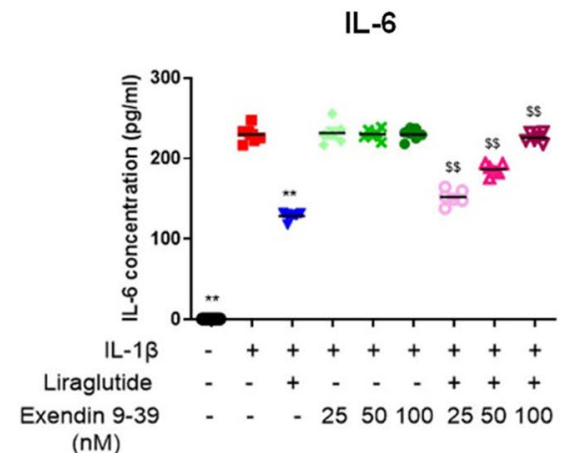

b

RAW 264.7 murine macrophages cell line
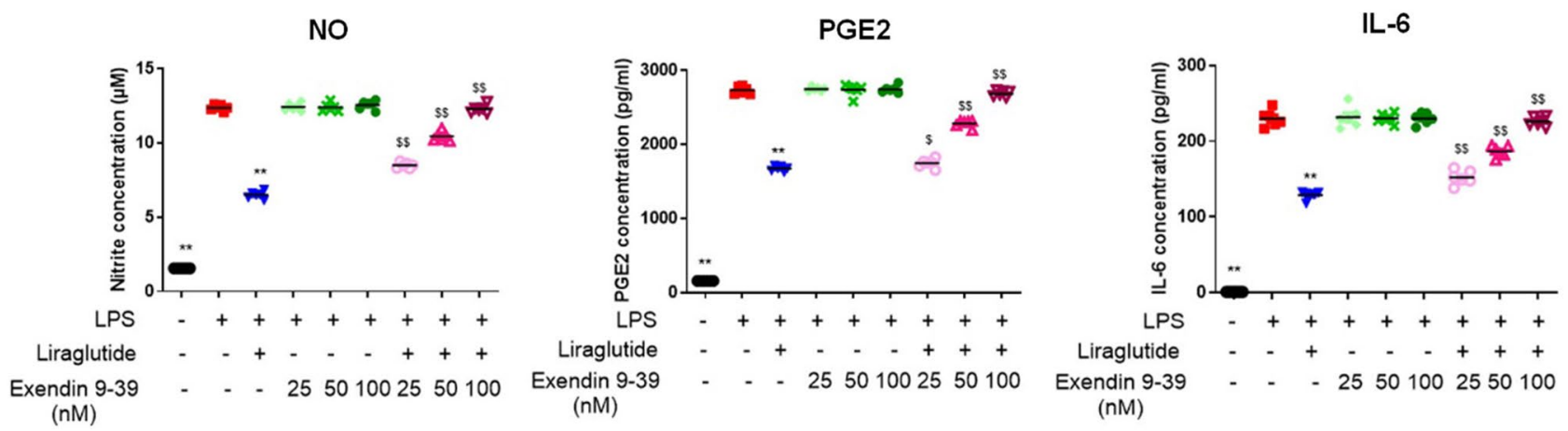

Figure 6. Anti-inflammatory effects of liraglutide are mediated by the GLP-1 receptor pathway. Primary cultured murine articular chondrocytes were incubated with $2 \mathrm{ng} / \mathrm{mL}$ IL-1 $\beta$ (a) and RAW 264.7, which were incubated with $100 \mathrm{ng} / \mathrm{mL}$ LPS (b) and co-treated with $50 \mathrm{nM}$ of liraglutide or co-treated with three doses of exendin fragment 9-39 (25 nM, $50 \mathrm{nM}$, and $100 \mathrm{nM})$ for $24 \mathrm{~h}(\mathrm{n}=6)$. Nitrite, PGE 2 , and IL-6 concentration of the supernatant was determined. Statistical analysis: Mean \pm SEM, Mann-Whitney test with sequential strategy, ${ }^{\star *} p<0.01$, versus stimulated control (IL-1 $\beta$ or LPS) and ${ }^{\$} p<0.05,{ }^{\$ \$} p<0.01$, versus IL- $1 \beta+$ liraglutide or LPS + liraglutide group.

secretion, $54 \mathrm{nM}$ [49-59 nM] for PGE 2 secretion, and $41 \mathrm{nM}$ [37-45 nM] for IL-6 secretion (Fig. 5a). Similarly, liraglutide treatment significantly reduced LPS-induced upregulation of $I l-6$, Cox2, and Tnf- $\alpha$ in RAW 264.7 cells ( $p=0.0286$ compared to LPS group) in a dose-dependent manner (Fig. 5b, Supplemental Table 1).

Macrophage polarization is subject to environmental cues. To test whether liraglutide can reverse the inflammatory phenotype of macrophages, LPS-stimulated RAW 264.7 cells were exposed to three increasing doses of liraglutide. Results showed that there was a downregulation in the expression of M1 pro-inflammatory macrophage-related genes, including $M c p-1$ and $C d 38$ (Fig. 5c). Conversely, there was an upregulation in the expression of Erg-2, a M2 phenotype-associated gene. These results indicated that liraglutide promoted macrophage repolarization from the pro-inflammatory M1 to the anti-inflammatory M2 phenotype in vitro, and that liraglutide could prevent or resolve inflammation at the tissue level.

The anti-inflammatory effect of liraglutide is mediated by the GLP-1 receptor signaling pathway. To determine whether GLP-1R was the sole target and mediator of the anti-inflammatory effects of liraglutide, we performed a functional competition assay using exendin 9-39, a GLP-1R competitive antagonist, in both chondrocytes and macrophages (Fig. 6). The chondrocytes and macrophages were exposed to liraglutide and increasing doses of exendin 9-39. The results showed that exendin 9-39 restored nitrite, $\mathrm{PGE}_{2}$, and IL-6 secretion in liraglutide pretreated chondrocytes and macrophages in a dose-dependent manner $(p=0.0022$ compared with IL- $1 \beta+$ liraglutide group and $p=0.026$ or $p=0.0022$ compared with LPS + liraglutide group). Treatment with $100 \mathrm{nM}$ of exendin 9-39 completely reversed the anti-inflammatory effect obtained with $50 \mathrm{nM}$ $\left(\mathrm{IC}_{50}\right.$ value) liraglutide. These data suggest that GLP-1R is the primary target of liraglutide in both chondrocytes and macrophages and that the anti-inflammatory effect of liraglutide is mediated by the GLP-1 receptor in these cell types (Fig. 6a,b).

Liraglutide exerts anti-catabolic effect in murine primary chondrocytes in vitro. To explore the inhibitory effect of liraglutide on the production of catabolic mediators, IL-1 $\beta$-stimulated chondrocytes were cultured with $2 \mathrm{ng} / \mathrm{mL}$ of IL- $1 \beta$ and 10 ascending doses $(6.6,13.3,26.6,53.1,106.3,212.5,425,850 \mathrm{nM}, 1.7$ and $3.4 \mu \mathrm{M}$ ) of liraglutide for $24 \mathrm{~h}$ for $\mathrm{IC}_{50}$ determination. The calculated $\mathrm{IC}_{50}$ was $56 \mathrm{nM}$ with confidence interval [52-62 nM] for MMP-3 secretion, $58 \mathrm{nM}$ [53-64 nM] for MMP-13 secretion, and $45 \mathrm{nM}$ [31-67 nM] for GAG 
a

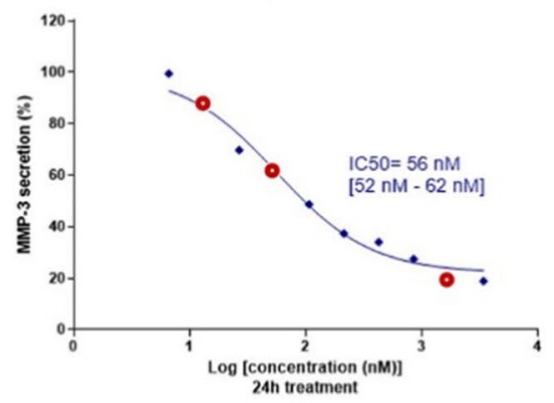

b
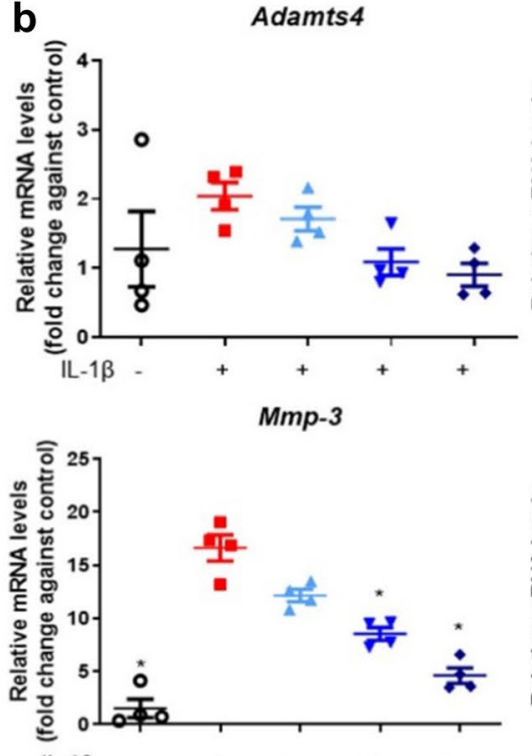

IL-1 $\beta$

- Vehicle

- IL-1 1 2 $\mathrm{ng} / \mathrm{ml}$
A IL-1ß $2 \mathrm{ng} / \mathrm{ml}+$ Liraglutide $13.3 \mathrm{nM}$

v IL-1ß $2 \mathrm{ng} / \mathrm{ml}+$ Liraglutide $53.1 \mathrm{nM}$
MMP-13

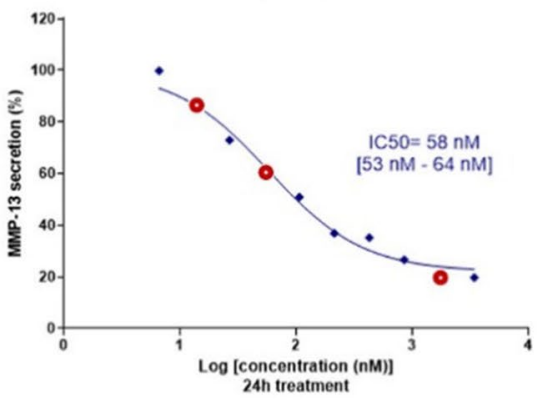

Adamts5
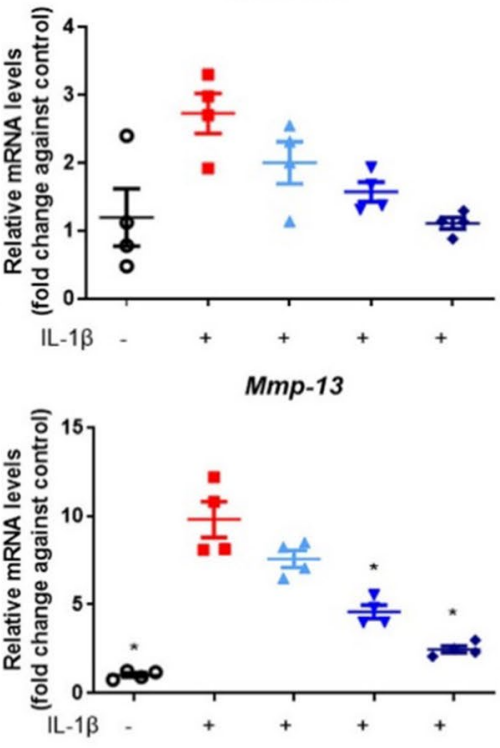

- IL-1ß $2 \mathrm{ng} / \mathrm{ml}+$ Liraglutide $1700 \mathrm{nM}$
rGAG

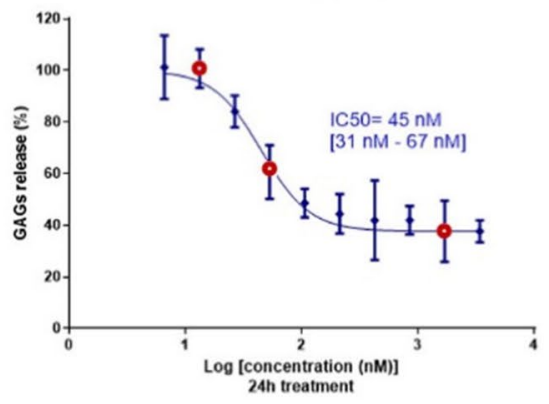

C

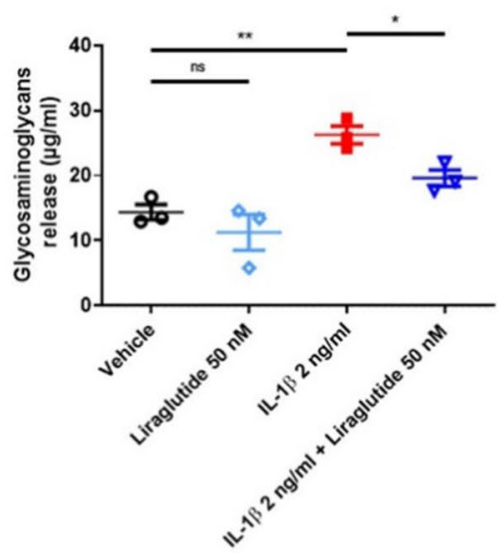

Figure 7. Anti-catabolic effects of liraglutide on murine primary chondrocytes. Murine primary chondrocytes were co-treated with $2 \mathrm{ng} / \mathrm{mL}$ of IL- $1 \beta$ and 10 doses of liraglutide $(6.6,13.3,26.6,53.1,106.3,212.5,425$, $850 \mathrm{nM}, 1.7$ and $3.4 \mu \mathrm{M})$ for $24 \mathrm{~h}(\mathrm{n}=4)$. (a) The inhibition rate of liraglutide in murine primary chondrocytes was analyzed using GraphPad Prism 9.0 and the $\mathrm{IC}_{50}$ values were determined. The expression of MMP-3, MMP13, and GAG (rGAG) was detected in the cell supernatant. (b) Relative mRNA expression of Adamts4, Adamts5, $M m p-3$, and $M m p-13$ in murine primary chondrocytes co-treated with IL- $1 \beta$ and liraglutide (13.3 nM, $53.1 \mathrm{nM}$, and $1700 \mathrm{nM})$ or vehicle for $24 \mathrm{~h}(\mathrm{n}=4)$. (c) Murine primary chondrocytes were stimulated with $2 \mathrm{ng} / \mathrm{mL}$ of IL-1 $\beta$ and co-treated with $50 \mathrm{nM}$ of liraglutide for $72 \mathrm{~h}(\mathrm{n}=3)$. The concentration of glycosaminoglycans was determined in the culture supernatant using the GAG assay. Statistical analysis: Mean \pm SEM, Mann-Whitney test with sequential strategy, ${ }^{*} p<0.05,{ }^{* *} p<0.01$, versus stimulated control (IL-1 $\beta$ alone). $n s$ non-statistical.

secreted from extracellular matrix (Fig. 7a). Moreover, the effect of three selected doses (13.3 nM, $53.1 \mathrm{nM}$, and $1700 \mathrm{nM}$ ) of liraglutide on Adamts4, Adamts5, Mmp-3, and Mmp-13 expression in IL- $1 \beta$-stimulated chondrocytes was evaluated. Results showed that liraglutide treatment significantly reduced $(p=0.0286)$ IL- $1 \beta$-induced expression of Adamts4, Adamts5, Mmp-3, and Mmp-13 in a dose-dependent manner compared with the IL- $1 \beta$ group (Fig. 7b). Furthermore, to explore the long-term anti-degradative effect of liraglutide, IL-1 $\beta$-stimulated chondrocytes were cultured with $2 \mathrm{ng} / \mathrm{mL}$ of IL- $1 \beta$ and $50 \mathrm{nM}$ of liraglutide for $72 \mathrm{~h}$. Results showed that liraglutide treatment $(50 \mathrm{nM})$ significantly reversed IL- $1 \beta$-induced increase in GAG release from the extracellular matrix of the chondrocytes ( $p=0.0227$ compared with IL-1 $\beta$ alone) (Fig. $7 \mathrm{c}$ ).

Overall, these results indicated that liraglutide may attenuate cartilage degradation in the long term via anticatabolic effect, as demonstrated in vitro.

\section{Discussion}

Repurposing clinically used drugs is an important strategy in drug discovery and could reduce cost and development timelines due to prior availability of safety and toxicity information ${ }^{27,28}$. The GLP-1/GLP-1R axis has been extensively studied in the context of diabetes. The GLP-1 analog liraglutide, a modified human GLP-1(7-37) with a longer half-life ${ }^{29}$, is administered systemically in patients with type II diabetes (commercial name Victoza) 
and obesity (commercial name Saxenda ${ }^{\circ}$ ). Recently, liraglutide has gained increasing attention owing to its antiinflammatory activities in age-related diseases ${ }^{30}$.

In the present study, it was observed that the liraglutide/GLP-1R axis acts in chondrocytes and macrophages to decrease inflammation and catabolism in vitro, which was confirmed by pain alleviation in vivo in mouse OA inflammatory pain model. Moreover, the expression of GLP-1R in OA patients indicated GLP-1R as a potential therapeutic target for OA treatment. The intra-articular injection of liraglutide for OA treatment could lead to drug repurposing.

GLP-1Rs are widely distributed in several cells, such as islet cells (especially $\beta$-cells of the pancreas), endothelial cells, neurons, adipocytes, keratinocytes, lymphocytes, hepatocytes, smooth muscle cells, myocytes, Brunner's glands in the duodenum, and myenteric plexus neurons in the gut ${ }^{31-34}$. These pleiotropic expressions indicate that GLP-1 and its drugs can exert extra-pancreatic functions. Recently, the expression of the GLP-1 receptor has been reported in the knee joint of rat cartilage ${ }^{35}$ and in chondrocytes from mouse tracheal cartilage ${ }^{36}$, but has never been described in OA patients or in mouse articular cartilage and synovial membrane. However, the results of the present study showed that GLP-1 receptor was expressed in the cartilage and synovial membrane of both OA human and OA and non-OA mice.

Recently, Chen et al. showed that the activation of GLP-1R by liraglutide could protect chondrocytes against endoplasmic reticulum (ER) stress, apoptosis, and inflammation by decreasing the release of inflammatory mediators ${ }^{35}$ in a surgically induced rat model of OA knee. Additionally, Que et al. demonstrated that the antiinflammatory effects of liraglutide act through the activation of the PKA/CREB pathway in a rat model of OA $\mathrm{knee}^{37}$. However, the potential analgesic effect of liraglutide is yet to be reported.

Pain, the major symptom of OA, is triggered by peripheral and central changes within the pain pathways. The mechanisms of pain in OA are complex and not yet well understood. It does not seem to be entirely driven by tissue damage, as highlighted by the discordance between pain and structural changes in OA joints ${ }^{38}$. Cartilage itself is not innervated, but nociceptors are present in surrounding tissues, such as the synovium and joint capsule, subchondral bone, periosteum, meniscus, and ligaments ${ }^{39}$. GLP-1R is expressed in dorsal root ganglion (DRG) neurons ${ }^{40}$ and could explain the potential direct effect on pain in OA since DRG neuron endings are present in the synovium ${ }^{41}$.

To explore the potential effect of liraglutide on OA-associated pain, MIA OA mice models were developed. This model has become a standard for modelling joint disruption in inflammatory OA in rodents. MIA injection to the knee joint leads to the progressive disruption of cartilage, which is associated with the development of pain-like behavior that mimics that of human OA. Thus, the MIA OA model is appropriate for studying the pharmacological effects of new drugs that can act on OA-related pain ${ }^{42,43}$. The findings of the present study indicated a dose-dependent analgesic effect of liraglutide in MIA OA mice model, with better results than dexamethasone (positive control treatment).

Local action seems to be paramount in sustaining an analgesic effect. In humans suffering from obesity, daily systemic injections of liraglutide did not ameliorate OA-related pain, probably because of poor access and hence poor local concentrations of liraglutide in the knee joint ${ }^{44}$.

To explain the analgesic effects of liraglutide, we hypothesized that its anti-inflammatory properties could play a central role. Recently, low-grade inflammation and innate immunity have been increasingly implicated in OA pathogenesis ${ }^{13,45}$. Synovial inflammation is associated with pain sensitization in patients with knee $\mathrm{OA}^{46}$, suggesting a role of pro-inflammatory mediators in this process ${ }^{39}$. Cytokines have been shown to sensitize knee joint nociceptors to mechanical stimulation in rats ${ }^{47}$. Moreover, inflammatory mediators can stimulate the distal terminals of DRG neurons, which in turn transmit sensory information to the central nervous system.

In vivo, we found that liraglutide improved the severity of synovitis in the MIA mouse model. Moreover, we conducted in vitro experiments on two murine cell types (chondrocytes and macrophages), reflecting two crucial cell types present in the knee joint. Immunohistochemical staining indicated the presence of GLP-1R at the membrane and cytoplasmic levels in both chondrocytes and macrophages. Additionally, inflammatory molecules produced by chondrocytes in response to IL- $1 \beta$ included PGE $_{2}$ and cyclooxygenase (Cox-2). Moreover, IL- $1 \beta$ can induce the accumulation of reactive oxygen species (ROS), through the expression of $i$ Nos. Furthermore, the results of the present study showed that liraglutide induced a dose-dependent anti-inflammatory effects in murine primary chondrocytes. Recently, Chen et al. demonstrated the anti-apoptotic and anti-inflammatory effects of GLP-1R activation in rat chondrocytes. Regulation of PI3K/Akt/ER stress is closely involved in the protective effects of GLP-1 $\mathrm{R}^{35}$.

Although fewer synovial macrophages are present in OA than in rheumatoid arthritis, they are crucial for the production of pro-inflammatory cytokines, such as IL-6 and TNF- $\alpha^{11}$. Previous studies have shown that selective depletion of synovial macrophages during experimental OA largely reduces cartilage damage and osteophyte formation, which are two major hallmarks of $\mathrm{OA}^{48}$. In LPS-stimulated macrophages, liraglutide significantly decreased Il-6, Cox2, and Tnf- $\alpha$ expression levels as well as PGE 2 , NO, and IL- 6 concentration.

Furthermore, macrophage polarization may play a role in OA progression. Indeed, a higher ratio of M1/ M2 was observed in synovial fluid from OA knee compared with normal knees, and the ratio was significantly correlated with the Kellgren-Lawrence grade ${ }^{49}$. However, it should be noted that macrophage phenotype plasticity in response to environmental conditions provides a broad spectrum of possibilities depending on their interactions ${ }^{50}$; thus, the classification of macrophages into the M1/M2 subtype is restrictive. In the present study, liraglutide promoted macrophage repolarization from M1 to M2 anti-inflammatory macrophage subtype in vitro. Thus, these results suggest that liraglutide could reduce, at least in part, the secretion of pro-inflammatory cytokines in OA synovium by stimulating macrophages and polarization toward an M2 anti-inflammatory phenotype, leading to a decrease in OA-induced joint destruction. However, these in vitro results on macrophage phenotype switch are still hypothetical and need to be tested in vivo. The effect of liraglutide on macrophage polarization has been verified in other tissues. Swada et al. recently revealed that liraglutide ameliorates the 
development of periodontitis, which was demonstrated by a decrease in periodontitis-induced inflammation, decrease in M1 macrophages in the gingiva, and alveolar bone loss with decreased osteoclast formation ${ }^{51}$. Moreover, Wan et al. demonstrated the effect of GLP-1 on macrophage activation, which contributed to M2 polarization and secretion of anti-inflammatory factors in RAW 264.7 cells $^{52}$.

Previous studies have demonstrated that the effect of GLP-1 involve both GLP-1R-dependent and-independent pathways ${ }^{53,54}$. To further investigate the anti-inflammatory effects of liraglutide and determine if the GLP-1R signaling pathway is involved in its anti-inflammatory effects, exendin fragment 9-39, a competitive GLP-1R antagonist was added to the incubation. The results of the present study demonstrated that exendin 9-39 at $100 \mathrm{nM}$ completely blocked the anti-inflammatory effect of $50 \mathrm{nM}$ liraglutide treatment. These data demonstrated that GLP-1R is the primary target of liraglutide in chondrocytes and macrophages and that the anti-inflammatory effect of liraglutide was mediated by the GLP-1 receptor pathway in both chondrocytes and macrophages. However, to further validate these results, the relationship between GLP-1 content and GLP-1R expression in response to liraglutide should be examined in future studies.

Targeting cartilage breakdown by reducing catabolic enzyme activity is an important strategy for treating or modify the course of $\mathrm{OA}^{55}$. Liraglutide treatment in chondrocytes significantly reduced IL- $1 \beta$-induced expression of Adamts4, Adamts5, Mmp-3 and Mmp-13 in a dose-dependent manner, leading to a decrease in MMP-3, MMP-13 and soluble GAG release by chondrocytes and macrophages. Taken together with its analgesic effects, these results indicated that liraglutide may attenuate cartilage degradation by inhibiting the expression of catabolic genes and protein in inflamed chondrocytes, as demonstrated in vitro. However, the findings of this study is limited by the small number of experiments performed, thus necessitating future studies to validate the findings of the present study. To validate this structural effect, it will be necessary to use an animal OA rodent model, such as the surgical destabilization of the medial meniscus (DMM), which is a more acceptable and suitable model to study the effects of liraglutide on cartilage degradation ${ }^{56}$.

Moreover, metabolic disturbances, such as obesity or diabetes mellitus, are associated with osteoarthritis, but data on the link between OA and lipid disturbances remain conflicting. A meta-analysis demonstrated an association between OA and dyslipidemia, indicating the role of metabolic disturbances in OA pathophysiology ${ }^{57}$. These results reinforce the concept of metabolic syndrome-associated OA phenotype. Liraglutide, like either approved GLP-1 drugs, has been shown to reduce weight, glucose and lipids in patients with Type 2 Diabetes Mellitus but also patients with overweight and obesity. GLP-1 signals through receptors primarily in the pancreas, gut and brain to increase insulin signalling and reduce food intake ${ }^{58}$. An extensive study of these metabolic changes, such as the effect of weight and glucose change on OA pain score, in response to liraglutide treatment is necessary. However, the present study is unique because it focused on the local effects of intra-articular injection of liraglutide and any potentially induce transient metabolic changes.

Disease-modifying osteoarthritis drugs (DMOADs) are a class of agents that target key tissues involved in $\mathrm{OA}$, and according to FDA guidelines, they must prevent structural progression and improve symptoms ${ }^{5,59}$. Currently, no DMOADs have been approved for use, but a number of potential therapies are in clinical trials ${ }^{60}$. However, all drugs in development face the challenge of showing both analgesic and structural properties ${ }^{61-64}$. Owing to its analgesic, anti-inflammatory, and anti-catabolic activities, liraglutide constitutes a new potential DMOAD for clinical development.

Overall, the findings of the present study demonstrated that intra-articular stimulation of the liraglutide/ GLP-1R axis has analgesic effects in vivo, which was probably due to the anti-inflammatory and anti-catabolic effects of liraglutide in vivo and in vitro, as demonstrated in vitro in macrophages and chondrocytes. Owing to its integrated mechanism of action on relevant cell types and the amelioration of OA symptoms, liraglutide could be a potential DMOAD for treating OA.

\section{Methods}

Chemicals and reagents. The GLP-1 analog, liraglutide, was purchased from Hybio Pharmaceuticals (Shenzhen, China) and Novo Nordisk (Bagsværd, Denmark). Dexamethasone was purchased from Mylan (Paris, France). Primary rabbit polyclonal immunoglobulin G (IgG) anti-GLP-1R antibody was purchased from Novus Biologicals (NBP1-97308, Centennial, Colo., USA). ImmPRESS horseradish peroxidase (HRP) goat antirabbit IgG polymer detection kit, BLOXALL, VectaFluor kit with anti-rabbit IgG Dylight 488 antibody, and VECTASHIELD $^{\circ}$ Antifade mounting medium (4',6-diamidino-2-phenylindole-DAPI) were purchased from Vector Laboratories (Burlingame, CA, USA). The 3,3'-diaminobenzidine (DAB) substrate kit was purchased from Cell Signaling Technology (Leiden, Netherlands). IL-1 $\beta$ was obtained from PeproTech (Neuilly-sur-Seine, France). Collagenase D was purchased from Roche (Basel, Switzerland), Griess reagent system was purchased from Promega (Charbonnière-les-Bains, France), mouse PGE2, IL-6, MMP-3 enzyme linked immunosorbent assay (ELISA), and LDH assay kits were purchased from Abcam (Cambridge, United Kingdom). Mouse ELISA kit for MMP-13 was purchased from Cloud-Clone Corp. (Katy, TX, USA), and glycosaminoglycans (GAG) assay kit was purchased from Chondrex (Redmond, Washington, USA). All culture media and chemicals, including exendin 9-39, were purchased from Sigma-Aldrich (St. Louis, MO, USA) unless otherwise stated.

Human samples. Human OA samples were isolated from the knees of six patients of different age (three women and three men; 68 to 83 years old for men, 71 to 83 years old for women) with varying osteoarthritis severity (Mankin score: 1.5-14 out of 14), undergoing joint arthroplasty at Assistance public-Hôpitaux de Paris (AP-HP) Saint-Antoine Hospital (Paris, France) ${ }^{65}$. For cartilage, $10 \mathrm{~mm}^{2}$ plugs of human OA cartilage and subchondral bone were obtained. Each plug included the entire cartilage layer and a piece of subchondral bone below the cartilage. Samples were fixed in $3.7 \%$ paraformaldehyde (PFA) at $4^{\circ} \mathrm{C}$ for $24 \mathrm{~h}$ and then decalcified in $500 \mathrm{mM}$ ethylenediaminetetraacetic acid (EDTA) ( $\mathrm{pH} 7.4$, VWR, Pennsylvania, USA ${ }^{66}$. Synovial membrane 
samples were dissected from adjacent adipose tissue and fixed in 3.7\% PFA for $24 \mathrm{~h}$. Cartilage or synovial membrane samples were embedded in paraffin, and $5 \mu \mathrm{m}$ sagittal sections were obtained using a Polycut E microtome.

Patients' data were anonymized, and informed consent for the use of tissue was obtained from each patient before surgery. Experiments using human samples were approved by French Institutional Review Board (Comité de Protection des Personnes, Paris Ile-de-France 5 and Commission Nationale de l'Informatique et des Libertés). All experiments were performed in accordance with relevant guidelines and regulations.

Immunohistochemical staining. The presence of GLP-1R in OA human knee joint sections harvested following arthroplasty and non-OA mouse knee joint sections harvested following a mouse study was evaluated using immunohistochemical staining. The sections were deparaffinized and rehydrated. Following incubation at $60^{\circ} \mathrm{C}$ overnight in $10 \mathrm{mM}$ sodium citrate buffer ( $\mathrm{pH}$ 6.0) for antigen unmasking, BLOXALL blocking solution was added to inactivate endogenous peroxidase (10 min at room temperature (RT)). Slides were then blocked with Tris-buffered saline (TBS) $+0.05 \%$ Tween 20 with $5 \%$ non-fat milk and $3 \%$ BSA, followed by $2.5 \%$ normal goat serum. Tissue sections were incubated with human anti-GLP-1R primary antibody $(0.02 \mathrm{mg} / \mathrm{mL})$ at $4^{\circ} \mathrm{C}$ overnight and then with secondary goat ImmPRESS ${ }^{\circ}$ HRP anti-rabbit IgG antibody at room temperature for $30 \mathrm{~min}$. The staining was revealed using DAB chromogen solution. Hematoxylin was used to counterstain nuclei (20 s). Images were captured using a Zeiss Axioplan 2 imaging microscope and analyzed using ImageJ software.

Immunofluorescence staining. Primary murine chondrocytes and RAW 264.7 murine macrophage cell line were fixed with $3.7 \%$ PFA for $30 \mathrm{~min}$ at RT, permeabilized with TBS $+0.05 \%$ Tween 20 buffer for $30 \mathrm{~min}$ at RT, and blocked with 2.5\% normal horse serum for $20 \mathrm{~min}$ at RT. Next, cells were incubated with $0.01 \mathrm{mg} / \mathrm{mL}$ primary antibody and then with secondary VectaFluor anti-rabbit IgG Dylight 488 antibody for $30 \mathrm{~min}$ at RT. VECTASHIELD ${ }^{\circ}$ Antifade mounting medium with DAPI was used for microscopic observation. Images were captured using a spinning disk confocal microscope (CSU-W1, Nikon) and analyzed using ImageJ software.

Sodium monoiodoacetate (MIA) model of OA in mice. Animal handling was performed according to the guidelines of the Federation of European Laboratory Animal Science Associations (FELASA). All experiments involving animals were performed following approval from the Ethics Committee for Animal Experimentation of Nord-Pas de Calais Region (CEEA75, APAFIS\#2019012113002115), and in compliance with the Animal Research: Reporting of In Vivo Experiments (ARRIVE) guidelines.

Wild-type male C57Bl/6 mice (12 weeks of age) were purchased from Janvier Labs (Le Genest-Saint-Isle, France). For the short-term MIA model, 120 animals were divided into seven groups: saline/vehicle group $(n=19$ mice), MIA/vehicle group $(n=18)$, all MIA/liraglutide groups $(n=17)$, and dexamethasone group $(n=15)$. For the long-term MIA model, 49 animals were divided into four groups: saline/vehicle ( $\mathrm{n}=9$ mice), MIA/vehicle groups $(n=10)$, MIA/liraglutide $(n=10)$, or dexamethasone $(n=10)$.

Mice (20-35 g) were maintained in plastic ventilated cages in a pathogen-free environment $(12 \mathrm{~h} / 12 \mathrm{~h}$ light/ dark cycle, $21-24^{\circ} \mathrm{C}$ ) at Institut Pasteur de Lille, and had ad libitum access to water and food. Mice were anesthetized with isoflurane $\left(2-5 \%\right.$ in $\mathrm{O}_{2}$ ) before intra-articular (IA) injection. Osteoarthritis was induced in the right knee joint through a single IA injection of MIA $(0.75 \mathrm{mg} / 5 \mu \mathrm{L}$ sterile $0.9 \%$ saline, $\mathrm{pH} 7.4)$ on day 1 . The MIA dose was selected based on a previously published study ${ }^{42}$. Mice in the control group were injected with an equivalent volume of saline. Following MIA induction, mice were allocated into treatment groups based on the results of von Frey test, which was performed on day 2 for short-term MIA model or day 7 for long-term MIA model. Mice were tested to quantify nociceptive threshold on days 7 and 10 in the short-term model and on days 14,21, and 28 after injection of MIA or saline in the long-term model. Animals were sacrificed on day 11 (short-term model) or day 29 (long-term model).

For the MIA short-term study, mice received a single IA injection of liraglutide $(1,5,10$, or $20 \mu \mathrm{g})$ or empty vehicle $(5 \mu \mathrm{L})$ into the right knee on day 3 . In the long-term MIA study, mice were administered $20 \mu \mathrm{g}$ liraglutide or vehicle control once a week for 3 weeks on day 8, 15, and 22. Dexamethasone $(20 \mu \mathrm{g})$ was used in these two experiments as an anti-inflammatory positive control following the same regimen ( 1 injection at day 3 in the short-term and 3 injections once a week from day 8 in the long-term MIA model). All measurements were performed by two observers blinded to the treatment. Mice were observed throughout the duration of each experiment for signs of mortality and morbidity, and overall appearance (activity, general response to handling, touch, ruffled fur). Mice were weighed twice per week.

Pain assessment. Joint nociception was evaluated using the von Frey test (tactile allodynia). Mice were placed individually in transparent plexiglass chambers and acclimated for $15 \mathrm{~min}$. Paw withdrawal threshold was calculated from the average of two trials, using Semmes-Weinstein von Frey monofilaments with bending forces of $0.008,0.02,0.04,0.07,0.16,0.4,0.6$, and $1.0 \mathrm{~g}$ ) applied in ascending order to the plantar surface. The lowest force from the test that produced a response was considered the withdrawal threshold. All procedures and testing were performed in a blinded manner.

Histological analysis. After the short-term MIA study, mice were euthanized on day 11, and the entire knee joints were removed and fixed in $4 \%$ PFA at $4^{\circ} \mathrm{C}$ for $72 \mathrm{~h}$ and then decalcified with 14\% EDTA solution ( $\mathrm{pH}$ 7.4). After dehydration and embedding in paraffin, the tissues were cut into $5 \mu \mathrm{m}$ thick sections using a Polycut E microtome and mounted on slides. Tissue morphology was evaluated by hematoxylin and eosin staining $(\mathrm{H} \& \mathrm{E})$ to assess chronic synovitis according to the Krenn score. The scores were blindly assigned by two different examiners. 
Cell culture and treatments. The murine macrophage cell line RAW 264.7 (TIB-71, American Type Culture Collection, Manassas, VA, USA) was cultured at $37^{\circ} \mathrm{C}$ and under $5 \% \mathrm{CO}_{2}$ condition in Dulbecco's modified Eagle's medium (DMEM) containing $4.5 \mathrm{~g} / \mathrm{L}$ glucose, $10 \%$ fetal bovine serum (FBS), and 1\% penicillin-streptomycin (P/S) mixture. RAW 264.7 cells were plated and treated in starving medium (DMEM containing $1 \% \mathrm{P} / \mathrm{S}$ ). The macrophage cell line was used before passage 20 for all the experiments.

Murine primary chondrocytes were isolated from the knee articular cartilage of 6 days-old C57Bl/6 mice as previously described ${ }^{67}$. Cell morphology examination, Alcian blue and Safranin O staining, and qRT-PCR for chondrocyte markers (Sox9, Col2a1, and Acan) confirmed that the cells extracted from the heads, femoral condyles, and tibial plates of mice were differentiated chondrocytes. Chondrocytes were cultured 7 days before starvation (with $0.1 \%$ bovine serum albumin (BSA)) and treatments. Chondrocytes were used at passage 0 for all the experiments.

Murine primary chondrocytes or RAW 264.7 cells were stimulated with $2 \mathrm{ng} / \mathrm{mL}$ of IL-1 $\beta$ or $100 \mathrm{ng} / \mathrm{mL}$ of lipopolysaccharide (LPS), respectively, and co-treated with ascending concentrations of liraglutide $(6.6,13.3$, 26.6, 53.1, 106.3, 212.5, 425, $850 \mathrm{nM}, 1.7$ and $3.4 \mu \mathrm{M})$ at $37^{\circ} \mathrm{C}+5 \% \mathrm{CO}_{2}$ for $24 \mathrm{~h}$. Exendin fragment 9-39, a competitive antagonist of GLP-1R, was used at concentrations of $25 \mathrm{nM}, 50 \mathrm{nM}$, and $100 \mathrm{nM}$. For long-term in vitro study, murine primary chondrocytes were stimulated with $2 \mathrm{ng} / \mathrm{mL}$ IL- $1 \beta$ and treated with $50 \mathrm{nM}$ of liraglutide at $37^{\circ} \mathrm{C}$ and $5 \% \mathrm{CO}_{2}$ for $72 \mathrm{~h}$.

Quantification of inflammatory and degradation marker. Total mouse PGE, IL-6, MMP-3, and MMP-13 concentration of primary murine chondrocytes $(p 0)$ or murine macrophage cell line $(p+9)$ after $24 \mathrm{~h}$ of treatment was assayed in cell-free supernatants using an ELISA kit, according to the manufacturer's instructions. The nitrite content of primary murine chondrocytes $(p 0)$ and murine macrophage cell line $(p+9)$ after $24 \mathrm{~h}$ of treatment was determined by the Griess method ${ }^{68}$ using a Griess Reagent System kit according to the manufacturer's instructions. Glycosaminoglycans concentration of primary murine chondrocytes (p0) after 24 or $72 \mathrm{~h}$ of treatment was measured using GAG Assay kit, according to the manufacturer's instructions. Lactate dehydrogenase assay (LDH) was performed to confirm the non-cytotoxicity of liraglutide treatment on both cell types. No enzymatic treatment was performed prior to the protein assay. Concentrations were analyzed using a spectrophotometer and determined by comparison against a standard curve.

Real-time quantitative PCR. Total ribonucleic acid (RNA) was extracted from murine chondrocytes or macrophages using the ReliaPrep ${ }^{\text {Ta }}$ RNA Cell Miniprep System (Promega, Charbonnière-les-Bains, France). Thereafter, $1 \mu \mathrm{g}$ of total RNA was reverse-transcribed to cDNA using the Omniscript ${ }^{\circ}$ RT kit (Qiagen, Courtaboeuf, France). To detect messenger RNA (mRNA) expression levels of GLP-1R downstream target genes, quantitative reverse transcription real-time polymerase chain reaction (RT-qPCR) was performed using GoTaq qPCR Master mix. The relative expression of target genes was calculated and normalized to that of hypoxanthine phosphoribosyltransferase 1 (HPRT) using the Delta C(T) method. The sequences of primers used are listed in Supplementary Table 1 .

Statistical analysis and $\mathrm{IC}_{50} / \mathrm{EC}_{50}$ determination. For all studies, groups were compared to control groups (saline or vehicle). A total of 15-19 mice per group and 9-10 mice per group were used for short-term and long-term studies, respectively. The choice of the number of experiments was established by power analysis tests and previously published work ${ }^{42,69}$. The small number of experiments used for each part of the work can be considered a limitation. All quantitative data were presented as mean \pm standard error of mean (SEM). GraphPad software (version 9.0) was used to perform statistical analysis. Non-parametric Mann-Whitney (sequential strategy) or two-way ANOVA was used to analyze significant differences between groups, and statistical significance was set at $p<0.05$. The inhibition percentages were analyzed using GraphPad Prism 9.0 and the half maximal inhibitory concentration $\left(\mathrm{IC}_{50}\right)$ or half maximal effective concentration $\left(\mathrm{EC}_{50}\right)$ values were determined.

Received: 14 June 2021; Accepted: 11 January 2022

Published online: 28 January 2022

\section{References}

1. Robinson, W. H. et al. Low-grade inflammation as a key mediator of the pathogenesis of osteoarthritis. Nat. Rev. Rheumatol. 12, 580-592. https://doi.org/10.1038/nrrheum.2016.136 (2016).

2. Palazzo, C., Ravaud, J. F., Papelard, A., Ravaud, P. \& Poiraudeau, S. The burden of musculoskeletal conditions. PLoS ONE 9, e90633. https://doi.org/10.1371/journal.pone.0090633 (2014).

3. James, S. L. et al. Global, regional, and national incidence, prevalence, and years lived with disability for 354 diseases and injuries for 195 countries and territories, 1990-2017: A systematic analysis for the Global Burden of Disease Study 2017. Lancet 392, 1789-1858 (2018).

4. Gupta, S., Hawker, G. A., Laporte, A., Croxford, R. \& Coyte, P. C. The economic burden of disabling hip and knee osteoarthritis (OA) from the perspective of individuals living with this condition. Rheumatology (Oxford) 44, 1531-1537. https://doi.org/10. 1093/rheumatology/kei049 (2005).

5. Osteoarthritis: Structural Endpoints for the Development of Drugs, Devices, and Biological Products for Treatment Guidance for Industry, 6 (2018).

6. Oo, W. M., Little, C., Duong, V. \& Hunter, D. J. The development of disease-modifying therapies for osteoarthritis (DMOADs): The evidence to date. Drug Des. Dev. Ther. 15, 2921-2945. https://doi.org/10.2147/DDDT.S295224 (2021).

7. Little, C. B. \& Hunter, D. J. Post-traumatic osteoarthritis: From mouse models to clinical trials. Nat. Rev. Rheumatol. 9, 485-497. https://doi.org/10.1038/nrrheum.2013.72 (2013). 
8. Sellam, J. \& Berenbaum, F. The role of synovitis in pathophysiology and clinical symptoms of osteoarthritis. Nat. Rev. Rheumatol. 6, 625-635. https://doi.org/10.1038/nrrheum.2010.159 (2010).

9. Loeser, R. F., Goldring, S. R., Scanzello, C. R. \& Goldring, M. B. Osteoarthritis: A disease of the joint as an organ. Arthritis Rheum. 64, 1697-1707. https://doi.org/10.1002/art.34453 (2012).

10. Hunter, D. J. Pharmacologic therapy for osteoarthritis-The era of disease modification. Nat. Rev. Rheumatol. 7, 13-22. https:// doi.org/10.1038/nrrheum.2010.178 (2011).

11. Bondeson, J. et al. The role of synovial macrophages and macrophage-produced mediators in driving inflammatory and destructive responses in osteoarthritis. Arthritis Rheum. 62, 647-657. https://doi.org/10.1002/art.27290 (2010).

12. Manferdini, C. et al. Adipose stromal cells mediated switching of the pro-inflammatory profile of M1-like macrophages is facilitated by PGE2: In vitro evaluation. Osteoarthr. Cartil. 25, 1161-1171. https://doi.org/10.1016/j.joca.2017.01.011,Pubmed:28153 787 (2017).

13. Berenbaum, F. Osteoarthritis as an inflammatory disease (osteoarthritis is not osteoarthrosis!). Osteoarthr. Cartil. 21, 16-21. https://doi.org/10.1016/j.joca.2012.11.012 (2013).

14. Oren, T. W., Botolin, S., Williams, A., Bucknell, A. \& King, K. B. Arthroplasty in veterans: Analysis of cartilage, bone, serum, and synovial fluid reveals differences and similarities in osteoarthritis with and without comorbid diabetes. J. Rehabil. Res. Dev. 48, 1195-1210. https://doi.org/10.1682/jrrd.2010.09.0186 (2011).

15. Nah, S. S. et al. Effects of advanced glycation end products on the expression of COX-2, PGE2 and NO in human osteoarthritic chondrocytes. Rheumatology (Oxford) 47, 425-431. https://doi.org/10.1093/rheumatology/kem376 (2008).

16. Laiguillon, M. C. et al. Characterization of diabetic osteoarthritic cartilage and role of high glucose environment on chondrocyte activation: Toward pathophysiological delineation of diabetes mellitus-related osteoarthritis. Osteoarthr. Cartil. 23, 1513-1522. https://doi.org/10.1016/j.joca.2015.04.026 (2015).

17. Courties, A., Berenbaum, F. \& Sellam, J. The phenotypic approach to osteoarthritis: A look at metabolic syndrome-associated osteoarthritis. Joint Bone Spine 86, 725-730. https://doi.org/10.1016/j.jbspin.2018.12.005 (2019).

18. Lee, H. et al. TissueGene-C promotes an anti-inflammatory micro-environment in a rat monoiodoacetate model of osteoarthritis via polarization of M2 macrophages leading to pain relief and structural improvement. Inflammopharmacology 28, 1237-1252. https://doi.org/10.1007/s10787-020-00738-y (2020).

19. Iwai, T., Ito, S., Tanimitsu, K., Udagawa, S. \& Oka, J.-I. Glucagon-like peptide-1 inhibits LPS-induced IL-1 $\beta$ production in cultured rat astrocytes. Neurosci. Res. 55, 352-360. https://doi.org/10.1016/j.neures.2006.04.008 (2006).

20. Andersen, A., Lund, A., Knop, F. K. \& Vilsbøll, T. Glucagon-like peptide 1 in health and disease. Nat. Rev. Endocrinol. 14, 390-403. https://doi.org/10.1038/s41574-018-0016-2 (2018).

21. Orskov, C., Wettergren, A. \& Holst, J. J. Biological effects and metabolic rates of glucagonlike peptide-1 7-36 amide and glucagonlike peptide-1 7-37 in healthy subjects are indistinguishable. Diabetes 42, 658-661. https://doi.org/10.2337/diab.42.5.658 (1993).

22. Lee, Y. S. et al. Glucagon-like peptide-1 inhibits adipose tissue macrophage infiltration and inflammation in an obese mouse model of diabetes. Diabetologia 55, 2456-2468. https://doi.org/10.1007/s00125-012-2592-3 (2012).

23. Dobrian, A. D. et al. Dipeptidyl peptidase IV inhibitor sitagliptin reduces local inflammation in adipose tissue and in pancreatic islets of obese mice. Am. J. Physiol. Endocrinol. Metab. 300, E410-E421. https://doi.org/10.1152/ajpendo.00463.2010 (2011).

24. Parthsarathy, V. \& Hölscher, C. The type 2 diabetes drug liraglutide reduces chronic inflammation induced by irradiation in the mouse brain. Eur. J. Pharmacol. 700, 42-50. https://doi.org/10.1016/j.ejphar.2012.12.012 (2013).

25. Berenbaum, F et al. EP2890390 - Treatment of Osteoarthritis WITH Incretin Hormones or Analogues Thereof (2014).

26. Krenn, V. et al. Synovitis score: Discrimination between chronic low-grade and high-grade synovitis. Histopathology 49, 358-364. https://doi.org/10.1111/j.1365-2559.2006.02508.x (2006).

27. Cha, Y. et al. Drug repurposing from the perspective of pharmaceutical companies. Br. J. Pharmacol. 175, 168-180. https://doi. org/10.1111/bph.13798 (2018).

28. Pushpakom, S. et al. Drug repurposing: Progress, challenges and recommendations. Nat. Rev. Drug Discov. 18, 41-58. https://doi. org/10.1038/nrd.2018.168 (2019).

29. Schisano, B. et al. GLP-1 analogue, liraglutide protects human umbilical vein endothelial cells against high glucose induced endoplasmic reticulum stress. Regul. Pept. 174, 46-52. https://doi.org/10.1016/j.regpep.2011.11.008 (2012).

30. Hansen, H. H. et al. The GLP-1 receptor agonist liraglutide improves memory function and increases hippocampal CA1 neuronal numbers in a senescence-accelerated mouse model of Alzheimer's disease. J. Alzheimers Dis. 46, 877-888. https://doi.org/10.3233/ JAD-143090 (2015).

31. Körner, M., Stöckli, M., Waser, B. \& Reubi, J. C. GLP-1 receptor expression in human tumors and human normal tissues: Potential for in vivo targeting. J. Nucl. Med. 48, 736-743. https://doi.org/10.2967/jnumed.106.038679 (2007).

32. Amato, A., Baldassano, S., Liotta, R., Serio, R. \& Mulè, F. Exogenous glucagon-like peptide 1 reduces contractions in human colon circular muscle. J. Endocrinol. 221, 29-37. https://doi.org/10.1530/JOE-13-0525 (2014).

33. Bang-Berthelsen, C. H. et al. GLP-1 induces barrier protective expression in Brunner's glands and regulates colonic inflammation. Inflamm. Bowel Dis. 22, 2078-2097. https://doi.org/10.1097/MIB.0000000000000847 (2016).

34. Amato, A. et al. Peripheral motor action of glucagon-like peptide-1 through enteric neuronal receptors. Neurogastroenterol. Motil. 22, 664. https://doi.org/10.1111/j.1365-2982.2010.01476.x (2010).

35. Chen, J. et al. Glucagon-like peptide-1 receptor regulates endoplasmic reticulum stress-induced apoptosis and the associated inflammatory response in chondrocytes and the progression of osteoarthritis in rat. Cell Death Dis. 9, 212. https://doi.org/10. 1038/s41419-017-0217-y (2018).

36. Andersen, D. B. et al. Using a reporter mouse to map known and novel sites of GLP-1 receptor expression in peripheral tissues of male mice. Endocrinology 162, 246. https://doi.org/10.1210/endocr/bqaa246 (2021).

37 Que, Q. et al. The GLP-1 agonist, liraglutide, ameliorates inflammation through the activation of the PKA/CREB pathway in a rat model of knee osteoarthritis. J. Inflamm. (Lond.) 16, 13. https://doi.org/10.1186/s12950-019-0218-y (2019).

38. Bedson, J. \& Croft, P. R. The discordance between clinical and radiographic knee osteoarthritis: A systematic search and summary of the literature. BMC Musculoskelet. Disord. 9, 116. https://doi.org/10.1186/1471-2474-9-116 (2008).

39. Malfait, A. M. \& Schnitzer, T. J. Towards a mechanism-based approach to pain management in osteoarthritis. Nat. Rev. Rheumatol. 9, 654-664. https://doi.org/10.1038/nrrheum.2013.138 (2013).

40. Anand, U. et al. Glucagon-like peptide 1 receptor (GLP-1R) expression by nerve fibres in inflammatory bowel disease and functional effects in cultured neurons. PLoS ONE 13, e0198024. https://doi.org/10.1371/journal.pone.0198024 (2018).

41. Chakrabarti, S., Jadon, D. R., Bulmer, D. C. \& Smith, E. S. J. Human osteoarthritic synovial fluid increases excitability of mouse dorsal root ganglion sensory neurons: An in-vitro translational model to study arthritic pain. Rheumatology (Oxford) 59, 662-667. https://doi.org/10.1093/rheumatology/kez331 (2020).

42 Pitcher, T., Sousa-Valente, J. \& Malcangio, M. The monoiodoacetate model of osteoarthritis pain in the mouse. J. Vis. Exp. 111, 53746. https://doi.org/10.3791/53746 (2016).

43. Conaghan, P. G., Cook, A. D., Hamilton, J. A. \& Tak, P. P. Therapeutic options for targeting inflammatory osteoarthritis pain. Nat. Rev. Rheumatol. 15, 355-363. https://doi.org/10.1038/s41584-019-0221-y (2019).

44. Gudbergsen, H. et al. Liraglutide after diet-induced weight loss for pain and weight control in knee osteoarthritis: A randomized controlled trial. Am. J. Clin. Nutr. 113, 314-323. https://doi.org/10.1093/ajcn/nqaa328 (2021). 
45. Syx, D., Tran, P. B., Miller, R. E. \& Malfait, A. M. Peripheral mechanisms contributing to osteoarthritis pain. Curr. Rheumatol. Rep. 20, 9. https://doi.org/10.1007/s11926-018-0716-6 (2018).

46. Neogi, T. et al. Association of joint inflammation with pain sensitization in knee osteoarthritis: The multicenter osteoarthritis study. Arthritis Rheumatol. 68, 654-661. https://doi.org/10.1002/art.39488 (2016).

47. Richter, F. et al. Tumor necrosis factor causes persistent sensitization of joint nociceptors to mechanical stimuli in rats. Arthritis Rheum. 62, 3806-3814. https://doi.org/10.1002/art.27715 (2010).

48. Ambarus, C. A. et al. Systematic validation of specific phenotypic markers for in vitro polarized human macrophages. J. Immunol. Methods 375, 196-206. https://doi.org/10.1016/j.jim.2011.10.013 (2012).

49. Liu, B., Zhang, M., Zhao, J., Zheng, M. \& Yang, H. Imbalance of M1/M2 macrophages is linked to severity level of knee osteoarthritis. Exp. Ther. Med. 16, 5009-5014. https://doi.org/10.3892/etm.2018.6852 (2018).

50 Wood, M. J. et al. Macrophage proliferation distinguishes 2 subgroups of knee osteoarthritis patients. JCI Insight. https://doi.org/ 10.1172/jci.insight.125325 (2019).

51. Sawada, N. et al. Glucagon-like peptide-1 receptor agonist liraglutide ameliorates the development of periodontitis. J. Diabetes Res. 2020, 8843310. https://doi.org/10.1155/2020/8843310 (2020).

52 Wan, S. \& Sun, H. Glucagon-like peptide-1 modulates RAW264.7 macrophage polarization by interfering with the JNK/STAT3 signaling pathway. Exp. Ther. Med. 17, 3573-3579. https://doi.org/10.3892/etm.2019.7347 (2019).

53. Ban, K. et al. Cardioprotective and vasodilatory actions of glucagon-like peptide 1 receptor are mediated through both glucagonlike peptide 1 receptor-dependent and -independent pathways. Circulation 117, 2340-2350. https://doi.org/10.1161/CIRCULATIO NAHA.107.739938 (2008).

54. Ossum, A., van Deurs, U., Engstrøm, T., Jensen, J. S. \& Treiman, M. The cardioprotective and inotropic components of the postconditioning effects of GLP-1 and GLP-1(9-36)a in an isolated rat heart. Pharmacol. Res. 60, 411-417. https://doi.org/10.1016/j. phrs.2009.06.004 (2009).

55. Mueller, M. B. \& Tuan, R. S. Anabolic/catabolic balance in pathogenesis of osteoarthritis: Identifying molecular targets. $P M R$ 3(Supplement 1), S3-S11. https://doi.org/10.1016/j.pmrj.2011.05.009 (2011).

56. Glasson, S. S., Blanchet, T. J. \& Morris, E. A. The surgical destabilization of the medial meniscus (DMM) model of osteoarthritis in the 129/SvEv mouse. Osteoarthr. Cartil. 15, 1061-1069. https://doi.org/10.1016/j.joca.2007.03.006 (2007).

57. Baudart, P., Louati, K., Marcelli, C., Berenbaum, F. \& Sellam, J. Association between osteoarthritis and dyslipidaemia: A systematic literature review and meta-analysis. RMD Open 3, e000442. https://doi.org/10.1136/rmdopen-2017-000442 (2017).

58 Yanai,. Effects of liraglutide, a human glucagon-like peptide- 1 analog, on glucose/lipid metabolism, and adipocytokines in patients with Type 2 diabetes. J. J. Endocrinol. Metab. https://doi.org/10.4021/jem38w (2011).

59 Adams, J. D. Pain and inflammation. Curr. Med. Chem. 27, 1444-1445. https://doi.org/10.2174/092986732709200327092413 (2020).

60. Ghouri, A. \& Conaghan, P. G. Update on novel pharmacological therapies for osteoarthritis. Ther. Adv. Musculoskelet. 11, X1986449 (2019).

61. Eckstein, F. et al. Intra-articular sprifermin reduces cartilage loss in addition to increasing cartilage gain independent of location in the femorotibial joint: Post-hoc analysis of a randomised, placebo-controlled phase II clinical trial. Ann. Rheum. Dis. 79, 525-528. https://doi.org/10.1136/annrheumdis-2019-216453 (2020).

62. Deshmukh, V. et al. A small-molecule inhibitor of the Wnt pathway (SM04690) as a potential disease modifying agent for the treatment of osteoarthritis of the knee. Osteoarthr. Cartil. 26, 18-27. https://doi.org/10.1016/j.joca.2017.08.015 (2018).

63. Yazici, Y. et al. Lorecivivint, a novel intraarticular CDC-like kinase 2 and dual-specificity tyrosine phosphorylation-regulated kinase 1A inhibitor and Wnt pathway modulator for the treatment of knee osteoarthritis: A phase II randomized trial. Arthritis Rheumatol. 72, 1694-1706. https://doi.org/10.1002/art.41315 (2020).

64 Grässel, S. \& Muschter, D. Recent advances in the treatment of osteoarthritis. F1000Res 9, 325. https://doi.org/10.12688/f1000 research.22115.1 (2020).

65. Mankin, H. J. Biochemical and metabolic aspects of osteoarthritis. Orthop. Clin. N. Am. 2, 19-31. https://doi.org/10.1016/S00305898(20)31137-8 (1971).

66. Courties, A. et al. Clearing method for 3-dimensional immunofluorescence of osteoarthritic subchondral human bone reveals peripheral cholinergic nerves. Sci. Rep. 10, 8852. https://doi.org/10.1038/s41598-020-65873-6 (2020).

67. Gosset, M., Berenbaum, F., Thirion, S. \& Jacques, C. Primary culture and phenotyping of murine chondrocytes. Nat. Protoc. 3, 1253-1260. https://doi.org/10.1038/nprot.2008.95 (2008).

68. Giustarini, D., Rossi, R., Milzani, A. \& Dalle-Donne, I. Nitrite and nitrate measurement by Griess reagent in human plasma: Evaluation of interferences and standardization. Methods Enzymol. 440, 361-380. https://doi.org/10.1016/S0076-6879(07)00823-3 (2008).

69 Ogbonna, A. C., Clark, A. K. \& Malcangio, M. Development of monosodium acetate-induced osteoarthritis and inflammatory pain in ageing mice. Age (Dordr.) 37, 9792. https://doi.org/10.1007/s11357-015-9792-y (2015).

\section{Acknowledgements}

The authors thank the Department of Orthopedic Surgery and Traumatology of Saint-Antoine Hospital for providing human OA tissues.

\section{Author contributions}

Conception and design: C. Meurot, C. Martin, L.S., J.B., C.B., R.R., K.B., F.B. Collection and assembly of data: C. Meurot, C. Martin, L.S., J.B., K.B., C.J., F.B. Analysis and interpretation: C. Meurot, C. Martin, L.S., J.B., R.R., K.B., C.J., F.B. Drafting of article: C. Meurot, C. Martin, K.B., C.J., F.B. Final approval of the article: C. Meurot, C. Martin, L.S., J.B., C.B., R.R., K.B., C.J., F.B. Provision of study materials or patients: L.S., R.R., C.J., F.B.

\section{Competing interests}

Dr. Berenbaum reports personal fees from AstraZeneca, Boehringer, Bone Therapeutics, CellProthera, Expanscience, Galapagos, Gilead, Grunenthal, GSK, Eli Lilly, Merck Sereno, MSD, Nordic Pharma, Nordic Bioscience, Novartis, Pfizer, Roche, Sandoz, Sanofi, Servier, UCB, Peptinov, 4P Pharma, grants from TRB Chemedica; In addition. Dr Berenbaum is the CEO of 4 Moving Biotech and chair of the scientific advisory board of $4 \mathrm{P}$ Pharma. Dr. Rattenbach is the CEO of 4P Pharma and the chairwoman of 4Moving Biotech. 4Moving Biotech and Sorbonne University own 2 patents for method of use of GLP1 analogs in the treatment of osteoarthritis (PCT/ FR2013/051998 and PCT/IB2018//059100). 


\section{Additional information}

Supplementary Information The online version contains supplementary material available at https://doi.org/ 10.1038/s41598-022-05323-7.

Correspondence and requests for materials should be addressed to F.B.

Reprints and permissions information is available at www.nature.com/reprints.

Publisher's note Springer Nature remains neutral with regard to jurisdictional claims in published maps and institutional affiliations.

(c) (1) Open Access This article is licensed under a Creative Commons Attribution 4.0 International cc) License, which permits use, sharing, adaptation, distribution and reproduction in any medium or format, as long as you give appropriate credit to the original author(s) and the source, provide a link to the Creative Commons licence, and indicate if changes were made. The images or other third party material in this article are included in the article's Creative Commons licence, unless indicated otherwise in a credit line to the material. If material is not included in the article's Creative Commons licence and your intended use is not permitted by statutory regulation or exceeds the permitted use, you will need to obtain permission directly from the copyright holder. To view a copy of this licence, visit http://creativecommons.org/licenses/by/4.0/.

(C) The Author(s) 2022 